\title{
Memory Effects and Scaling Laws in Slowly Driven Systems
}

\author{
N. Berglund and H. Kunz \\ Institut de Physique Théorique \\ Ecole Polytechnique Fédérale de Lausanne \\ PHB-Ecublens, CH-1015 Lausanne, Switzerland \\ e-mail: berglund@iptsg.epfl.ch, kunz@dpmail.epfl.ch
}

July 17, 1998

\begin{abstract}
This article deals with dynamical systems depending on a slowly varying parameter. We present several physical examples illustrating memory effects, such as metastability and hysteresis, which frequently appear in these systems. A mathematical theory is outlined, which allows to show existence of hysteresis cycles, and determine related scaling laws.
\end{abstract}

Key words: adiabatic theory, slow-fast systems, bifurcation theory, dynamic bifurcations, bifurcation delay, hysteresis, metastability, scaling laws

PACS numbers: 05.45.+b, 75.10.-d, 75.40.Gb, 75.60.-d 


\section{Introduction}

There exist many instances where the dynamics of a system depends on a parameter which varies slowly in time. This parameter is often controllable by the experimentalist, who can modify it at will. A well-known example of this situation is that of a ferromagnet on which is imposed a low frequency magnetic field. One can also think of chemical reactions occurring in a reactor in which the flux of the injected chemical substances is varying slowly, or the Couette-Taylor experiment in hydrodynamics where the speed of rotation of the inner cylinder is slowly modulated. In other circumstances, the parameter is not controllable, but certainly influences the dynamics of the system of interest. As examples of this situation we could mention the case of the impact of solar light on the thermal convection in the atmosphere, or the seasonal (or even climatic) effect on the dynamics of populations.

One of the most interesting phenomena observed in systems with an adiabatically varying parameter is the familiar one of hysteresis. Recently, there has been a renewal of interest in this old problem, both from a theoretical and an experimental point of view. Several authors [RKP, SE, JGRM, HL\&] have particularly analysed properties of the hysteresis cycle, such as its area, which appears to scale in a nontrivial way with the adiabatic parameter.

In this article, we concentrate on dynamical systems with a finite number of degrees of freedom, depending on a parameter in such a way that the system undergoes bifurcations when the parameter is considered to be static. The static (or "frozen") situation corresponds to measurements made, in principle during very long times, at successive fixed values of the parameter. We then ask what is happening when the parameter is varying slowly in time, instead of being kept fixed. This question is closely related to the opposite one: can the static bifurcation diagram be determined experimentally by varying the parameter slowly in time (a possible temptation for the impatient experimentalist)?

We have recently developed a coherent mathematical framework to deal with adiabatic systems, in particular to show existence of hysteresis cycles and determine their scaling laws Ber]. The purpose of this article is to explain these methods by illustrating them on a few concrete physical examples.

We begin, in Section 2, by presenting the most important features of onedimensional (1D) systems, which are illustrated by a few generic examples. We discuss in particular a simple geometric method to determine scaling laws near bifurcation points.

In Section 3, we use the Lorenz model to illustrate the phenomenon of bifurcation delay. When translated into the language of Rayleigh-Bénard (RB) convection, this phenomenon means that the slow and periodic variation of the temperature gradient in time leads to a delayed appearance of convection rolls and to hysteresis. The Lorenz model being a good approximation close to the instability threshold, since it contains two dominant modes of the bifurcation, this delay should be observable in the real RB convection. To explain the delay, we introduce two new methods especially designed for $n$-dimensional $(n \mathrm{D})$ systems: dynamic diagonalization and adiabatic manifolds. 
In Section 4, we present a simple mean field model for the dynamics of a ferromagnet in a slowly oscillating magnetic field. In the 1D case, we discuss the concept of dynamic phase transition introduced in [TO], and derive a scaling law for the hysteresis area. In the $2 \mathrm{D}$ case, we examine the effect of anisotropy on the mechanism of magnetization reversal and the shape of hysteresis cycles.

In Section 5, we discuss a simple mechanical system (which was introduced in [BK]), displaying chaotic instead of periodic hysteresis. This phenomenon depends only on a few qualitative features of the system, and should be observable in a larger class of nonlinear oscillators including inertia and involving a symmetry breaking bifurcation.

Finally, Section 6 is dedicated to some examples of the effect of eigenvalue crossings. These crossing give rise to an effective interaction between otherwise independent modes, which is essential in the sense that it cannot be eliminated by a change of variables. The interaction may, however, be delayed in certain cases.

Throughout this text, we use the following mathematical setting. The "frozen" dynamical system is supposed to be described by a family of ordinary differential equations

$$
\frac{\mathrm{d} x}{\mathrm{~d} t}=F(x, \lambda), \quad x \in \mathbb{R}^{n}, \quad \lambda \in \mathbb{R} .
$$

The associated adiabatic system is given by

$$
\frac{\mathrm{d} x}{\mathrm{~d} t}=F(x, \lambda(\varepsilon t))
$$

where $\lambda(\tau)$ is a given function, and $\varepsilon$ is the small adiabatic parameter. It is useful to introduce the slow time $\tau=\varepsilon t$, so that (2) can be rewritten as

$$
\varepsilon \frac{\mathrm{d} x}{\mathrm{~d} \tau}=F(x, \lambda(\tau))
$$

or, in short form, $\varepsilon \dot{x}=f(x, \tau)$. We denote by $\langle\cdot \mid \cdot\rangle$ the usual scalar product in $\mathbb{R}^{n}$ and by $\|\cdot\|$ the Euclidean norm.

There is a large literature on singular perturbed problems of this type. Results on linear systems can be found in [Wa]. For a review of results on dynamic bifurcations, see [Ben]. In particular, the phenomenon of bifurcation delay has been rigorously analysed in two important articles by Neishtadt Ne1, Ne2. Certain hysteresis phenomena in slow-fast systems similar to the Van der Pol equation have been analysed in $\mathbf{M K} \&$. Our geometric method to determine scaling exponents, as well as the procedures using dynamic diagonalization and adiabatic manifolds, however, are new to our knowledge. Here we only outline some essential ideas of these methods, detailed proofs can be found in [Ber]. 


\section{One-dimensional systems}

In this section, we will consider one-dimensional (1D) adiabatic equations of the form

$$
\varepsilon \dot{x}=F(x, \lambda(\tau))=f(x, \tau), \quad x, \tau \in \mathbb{R},
$$

where the dot denotes derivation with respect to $\tau$, and $f(x, \tau)$ is assumed to be an analytic real-valued function (weaker results as those stated below hold for differentiable functions).

The static bifurcation diagram of (4) is obtained by determining the solutions of $f(x, \tau)=0$, which are generically curves subdividing the plane into regions where $f$ is positive or negative. Let $x^{\star}(\tau)$ be such an equilibrium curve. The implicit function theorem tells us that if the linearization $a(\tau)=\partial_{x} f\left(x^{\star}(\tau), \tau\right)$ does not vanish, then $x^{\star}(\tau)$ is a smooth curve. It corresponds to stable solutions if $a(\tau)$ is negative, and to unstable ones of $a(\tau)$ is positive.

In such a situation, one can prove the existence of a particular solution $\bar{x}(\tau)$ of the adiabatic equation (4) tracking the curve $x^{\star}(\tau)$ at a distance of order $\varepsilon$ :

$$
\bar{x}(\tau)=x^{\star}(\tau)+\mathcal{O}(\varepsilon) .
$$

Moreover, this solution admits an asymptotic power series in $\varepsilon$, which does not converge in general, but admits, however, an optimal truncation at exponentially small order:

$$
\bar{x}(\tau)=x^{\star}(\tau)+\sum_{j=1}^{N(\varepsilon)} x_{j}(\tau) \varepsilon^{j}+\mathcal{O}\left(\mathrm{e}^{-1 / C \varepsilon}\right), \quad N(\varepsilon)=\mathcal{O}(1 / \varepsilon) .
$$

We call $\bar{x}(\tau)$ an adiabatic solution associated with the equilibrium branch $x^{\star}(\tau)$. Other solutions of (田) are attracted or repelled exponentially fast by adiabatic ones, and tend to switch between the neighborhoods of different equilibrium branches in a time of order $\varepsilon|\ln \varepsilon|$. As long as there are no bifurcation points, the solutions thus remain most of the time close to equilibria, and there is no hysteresis in the system.

Let us now consider the effect of bifurcations. At these special points, several equilibrium branches may meet, causing the solutions to choose between several possible directions, which is the basic mechanism of hysteresis. Moreover, equilibrium branches are in general no longer tracked at a distance of order $\varepsilon$, but at a distance scaling in some other, nontrivial way with $\varepsilon$, which we now show how to compute.

If the origin is a bifurcation point of $f$, we can write in some neighborhood

$$
f(x, \tau)=\sum_{n, m \geqslant 0} c_{n m} x^{n} \tau^{m}, \quad c_{00}=c_{10}=0 .
$$

Assume that $f(x, \tau)$ admits an equilibrium branch scaling as $x^{\star}(\tau) \approx|\tau|^{q}$ (we use this notation to indicate that $c_{-}|\tau|^{q} \leqslant x^{\star}(\tau) \leqslant c_{+}|\tau|^{q}$, where $c_{ \pm}$are positive constants independent of $\tau$ and $\varepsilon$ ). A standard result of bifurcation theory states that $-q$ is 

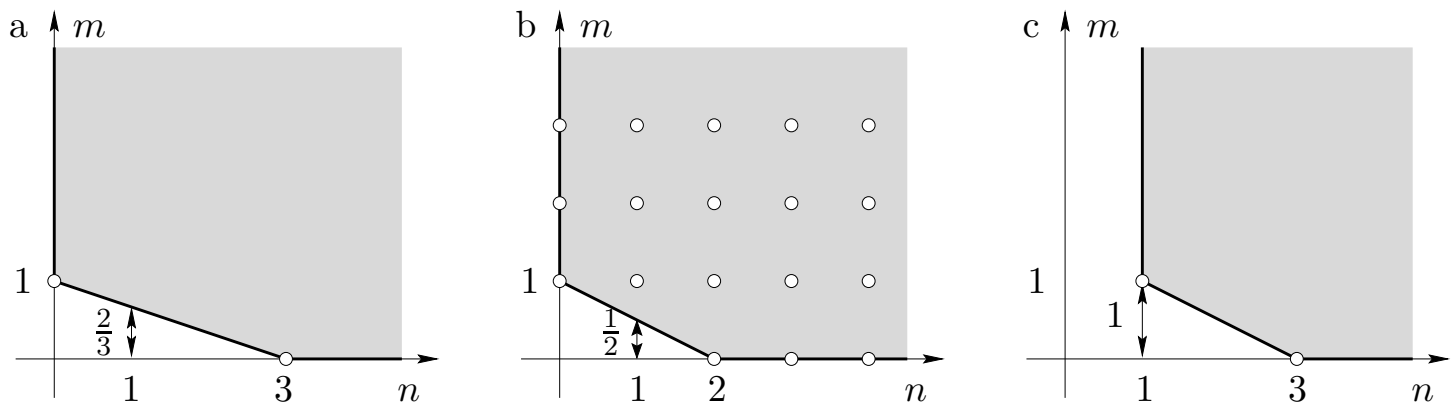

Figure 1. Newton's polygons for the most generic bifurcations discussed in the text. Dots mark points for which $c_{n m} \neq 0$. The slope of segments correspond to the possible exponents $q$ of equilibrium branches through the bifurcation point. The ordinate at 1 of these segments is the exponent $p$ of the linearization.

necessarily equal to the slope of a segment of Newton's polygon. This polygon is constructed as the convex envelope of the set of points $(n, m)$ such that $c_{n m} \neq$ 0 , completed by a horizontal and a vertical (Fig. 1). The linearization $a(\tau)=$ $\partial_{x} f\left(x^{\star}(\tau), \tau\right)$ scales generically as $|\tau|^{p}$, where $p$ is the ordinate at 1 of the tangent to Newton's polygon with slope $-q$.

These two easily determined numbers $p$ and $q$ are usually sufficient to characterize the scaling behaviour at leading order in $\varepsilon$. In fact, different behaviours take place in an inner region $|\tau| \leqslant \varepsilon^{1 / p+1}$ and in an outer region $|\tau| \geqslant \varepsilon^{1 / p+1}$. In particular, if $x^{\star}(\tau) \approx|\tau|^{q}$ is a decreasing stable branch arriving at the bifurcation point, above which $f$ is negative, one can show that

$$
\bar{x}(\tau)-x^{\star}(\tau) \approx \begin{cases}\varepsilon|\tau|^{q-p-1} & \text { for } \tau \leqslant-\varepsilon^{1 / p+1} \\ \varepsilon^{q / p+1} & \text { for }-\varepsilon^{1 / p+1} \leqslant \tau \leqslant 0 .\end{cases}
$$

Combining a local analysis around bifurcation points with a global analysis, which is usually easy in $1 \mathrm{D}$, one can determine the qualitative properties of dynamics. In particular, if $\lambda(\tau)$ is a periodic function, one can construct the Poincaré map (which is necessarily a monotonous function) in order to prove existence of hysteresis cycles and determine their scaling laws.

Let us illustrate this procedure on the simple model equation given by

$$
F(x, \lambda, \mu)=-\mu x-x^{3}+\lambda .
$$

Mathematically, this function is a generic two-parameter perturbation of the vector field $-x^{3}[\mathbf{H K}$. Physically, it describes the overdamped motion of a particle in a Ginzburg-Landau potential

$$
\Phi(x, \lambda, \mu)=\frac{1}{2} \mu x^{2}+\frac{1}{4} x^{4}-\lambda x,
$$

where $\mu=T-T_{\mathrm{c}}$ represents the difference between the temperature and its critical value, and $\lambda$ is an external field. The quartic potential described by the first two 
a

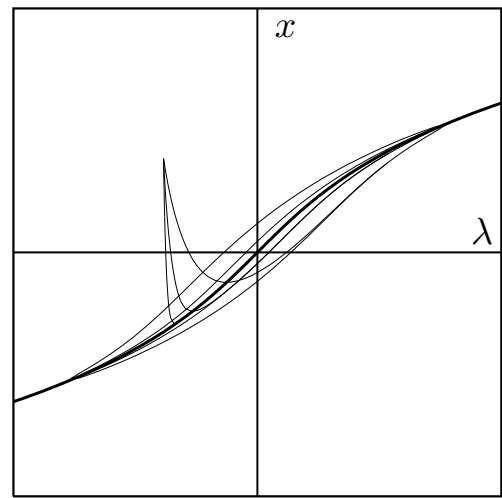

$\mathrm{b}$

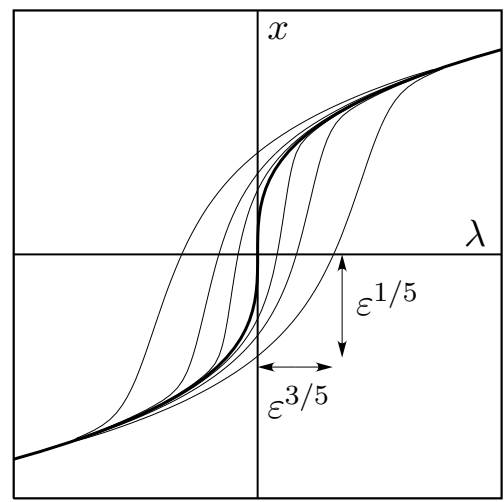

Figure 2. (a) Orbits, in the $(\lambda, x)$-plane, of the system $\varepsilon \dot{x}=-\mu x-x^{3}+\lambda(\tau)$, for $\mu=1$ and $\varepsilon=10^{-3 / 2}, 10^{-2}$ and $10^{-5 / 2}$. The solutions are attracted by a periodic orbit, enclosing an area of order $\varepsilon$. (b) Same as (a), but for $\mu=0$. At $\lambda=0$, the periodic orbit is at a distance of order $\varepsilon^{1 / 5}$ of the equilibrium point, and it encloses an area of order $\varepsilon^{4 / 5}$.

terms is fairly generic in physical systems presenting the symmetry $x \mapsto-x$, while the linear term is the simplest possible asymmetric perturbation.

We begin with the situation where $\mu=1$ is fixed, and $\lambda(\tau)=\sin \tau$ is slowly oscillating. The equation $F(x, \lambda, 1)=0$ admits a single, stable equilibrium branch $x^{\star}(\lambda)$, given implicitly by $x^{\star}(\lambda)^{3}+x^{\star}(\lambda)=\lambda$. All solutions are attracted by a periodic solution $\bar{x}(\tau)=x^{\star}(\lambda(\tau))+\mathcal{O}(\varepsilon)$, enclosing an area of order $\varepsilon$ (Fig. 2a). In the adiabatic limit $\varepsilon \rightarrow 0$, this area vanishes and there is no hysteresis.

If $\mu=0$ and $\lambda(\tau)=\sin \tau$, the unique equilibrium branch $x^{\star}(\lambda)=\lambda^{1 / 3}$ admits the origin as a bifurcation point. Using Newton's polygon or a direct calculation, we find that the exponents determining the scaling behaviour are $q=\frac{1}{3}$ and $p=\frac{2}{3}$ (Fig. 11a). The orbits are attracted by a periodic one, crossing the $x$-axis at a distance of order $\varepsilon^{1 / 5}$ from the origin, and enclosing an area

$$
\mathcal{A}(\varepsilon) \approx \varepsilon^{4 / 5}
$$

(Fig. 2b). The cycle still collapses with $x^{\star}(\lambda(\tau))$ in the adiabatic limit, but with a much slower rate. These exponents have been found in [HL\&, GBS] using other methods (which are less general than ours).

If $\mu=-1$ and $\lambda(\tau)=\sin \tau$, there are two bifurcation points at $\left( \pm \lambda_{\mathrm{c}}, \mp x_{\mathrm{c}}\right)$, where $\lambda_{\mathrm{c}}=\sqrt{4 / 27}$ and $x_{\mathrm{c}}=\sqrt{1 / 3}$. Two stable branches $x_{ \pm}^{\star}(\tau)$ and one unstable branch $x_{0}^{\star}(\tau)$ meet at these bifurcation points (Fig. Ba). Since they are crossed with nonzero velocity, Newton's polygon shows that the associated exponents are $q=p=\frac{1}{2}$ (Fig. [1]b). In fact, close to these points, the dynamics in translated coordinates is governed by the equation

$$
\varepsilon \dot{y}=-\tau-y^{2}+\text { higher order terms. }
$$

Solutions cross the $y$-axis at $y \approx \varepsilon^{1 / 3}$. Using the scaling $y=\varepsilon^{1 / 3} z$ and $\tau=\varepsilon^{2 / 3} \sigma$, one shows that $y$ remains of order $\varepsilon^{1 / 3}$ until a time $\tau^{\star} \approx \varepsilon^{2 / 3}$, and then quickly leaves 
a

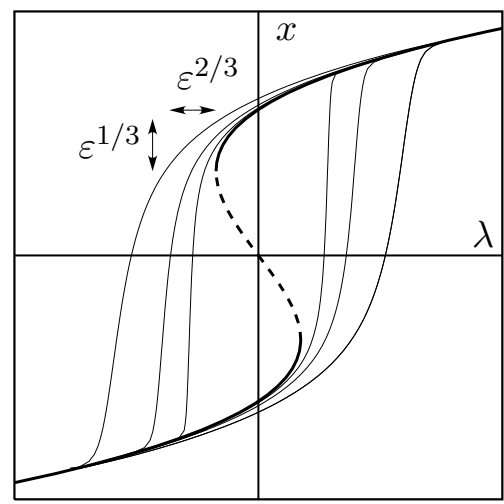

b

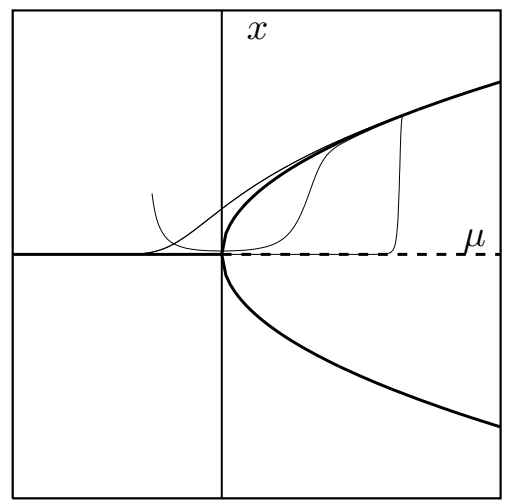

Figure 3. (a) Same as Fig. 2, for $\mu=-1$. The periodic orbit lies at a distance at most $\mathcal{O}\left(\varepsilon^{1 / 3}\right)$ from a limiting hysteresis cycle, composed of stable equilibrium branches and two vertical lines. The enclosed area is $\mathcal{A}(\varepsilon)=\mathcal{A}(0)+\mathcal{O}\left(\varepsilon^{2 / 3}\right)$. (b) Hysteresis cycle of the equation $\varepsilon \dot{x}=\mu(\tau) x-x^{3}$.

the bifurcation region to jump on the other stable branch. The orbits are attracted by a hysteresis cycle with area satisfying

$$
\mathcal{A}(\varepsilon)-\mathcal{A}(0) \approx \varepsilon^{2 / 3}
$$

where $\mathcal{A}(0)=\frac{3}{2}$ is the area situated between $x_{+}^{\star}(\lambda)$ and $x_{-}^{\star}(\lambda)$ for $-\lambda_{c}<\lambda<$ $\lambda_{\mathrm{c}}$. This time, the hysteretic behaviour persists in the adiabatic limit. The main contribution of order $\varepsilon^{2 / 3}$ to the excess area comes from the delayed jump. The scaling law (13) was also obtained in [JGRM using an exact solution of (12) (without the higher order terms).

As a final example, let us consider the situation where $\lambda=0$ and $\mu(\tau)$ is the varying parameter. The static bifurcation diagram displays a pitchfork bifurcation at the origin, involving the branches $x \equiv 0$ and $x= \pm \sqrt{\lambda}$, for which $q=\frac{1}{2}$ and $p=1$ (Fig. 1 $\mathrm{c}$ ). An important new phenomenon is bifurcation delay: near $x=0$, the dynamics is essentially governed by the linearized equation

$$
\varepsilon \dot{x}=\mu(\tau) x \quad \Rightarrow \quad x(\tau)=\exp \left[\frac{1}{\varepsilon} \int_{\tau_{0}}^{\tau} \mu(s) \mathrm{d} s\right] x\left(\tau_{0}\right)
$$

Starting at a time where $\mu<0, x(\tau)$ remains exponentially small as long as the integral in (14) is negative, which is true for a while beyond the instant where $\mu$ becomes positive. If the solution finally jumps on the stable branch and $\mu$ is decreased again, this branch is followed adiabatically, which leads to hysteresis (Fig. 3b). The area of the cycle follows the scaling law

$$
\mathcal{A}(\varepsilon)-\mathcal{A}(0) \approx \varepsilon^{3 / 4}
$$

where $\mathcal{A}(0)$ depends on the bifurcation delay time.

These examples indicate that one-dimensional systems are relatively well understood. In fact, our method to determine scaling laws is general, and quite straightforward to apply to other bifurcations than the special cases described here. Moreover, 
one can expect that when a mode of a larger system undergoes bifurcation, the dynamics of the associated adiabatic system will be governed by an effective 1D equation, describing the motion of this particular mode, which explains why the same scaling laws are observed for more complicated systems. The Lorenz model discussed in the next section illustrates this reduction of variables.

\section{Bifurcation delay in the Lorenz model}

Let us now turn to the behaviour of higher-dimensional systems

$$
\varepsilon \dot{x}=F(x, \lambda(\tau))=f(x, \tau), \quad x \in \mathbb{R}^{n}, \tau \in \mathbb{R} .
$$

Adiabatic solutions with an expansion of the form (6) still exist in the vicinity of hyperbolic equilibria, i.e., branches of equilibrium points around which the linearization of $f$ has no purely imaginary eigenvalues.

The behaviour of neighbouring solutions is far more complicated to analyse than in the $1 \mathrm{D}$ case. One can, however, obtain valuable informations by analysing the linearized system first, before dealing with nonlinear terms using appropriate tools such as invariant manifolds.

We illustrate these techniques on the Lorenz model with slowly varying temperature gradient $r(\tau)$ :

$$
\begin{aligned}
& \varepsilon \dot{x}_{1}=\sigma\left(x_{2}-x_{1}\right) \\
& \varepsilon \dot{x}_{2}=r(\tau) x_{1}-x_{2}-x_{1} x_{3} \\
& \varepsilon \dot{x}_{3}=-b x_{3}+x_{1} x_{2},
\end{aligned}
$$

where we assume that $b, \sigma>0$. This model has been introduced as an approximation to Rayleigh-Bénard convection, but also describes other systems such as lasers. It is well known that if $r \leqslant 1$ is fixed, the origin is a globally asymptotically stable fixed point, whereas when $r>1$, the origin is hyperbolic and two new equilibria $C_{ \pm}=$ $( \pm \sqrt{b(r-1)}, \pm \sqrt{b(r-1)}, r-1)$ appear, which correspond physically to convection rolls.

We will study this system when $r(\tau)$ is slowly oscillating around $r=1$, and stays well below the chaotic region. It can be written in compact form

$$
\varepsilon \dot{x}=A(\tau) x+b(x)
$$

where $b(x)$ is quadratic. Note that the identically zero function is a particular solution of this equation. The matrix $A(\tau)$ has three eigenvalues

$$
a_{1,2}(\tau)=-\frac{1}{2}(\sigma+1) \pm s(\tau), \quad a_{3}=-b, \quad s(\tau):=\frac{1}{2} \sqrt{(\sigma+1)^{2}+4 \sigma(r(\tau)-1)} .
$$

The eigenvalues $a_{2}$ and $a_{3}$ are always negative, while $a_{1}(\tau)$ has the same sign as $r(\tau)-1$. We thus expect that the motion will essentially follow the eigenspace of

$a_{1}(\tau)$. For this reason, we will construct a change of variables isolating this particular 
direction. To do this, we begin by searching a linear transformation which should diagonalize the linear part of (18). To this end, observe that if $S(\tau ; \varepsilon)$ is a matrix satisfying

$$
\varepsilon \dot{S}=A S-S D
$$

where $D(\tau ; \varepsilon)$ is diagonal, then the change of variables $x=S y$ transforms (18) into

$$
\varepsilon \dot{y}=D(\tau) y+S^{-1} b(S y) .
$$

The key point is that we can prove the existence of a bounded solution of (20), admitting asymptotic series

$$
\begin{aligned}
& S(\tau ; \varepsilon)=S_{0}(\tau)+\varepsilon S_{1}(\tau)+\varepsilon^{2} S_{2}(\tau)+\cdots \\
& D(\tau ; \varepsilon)=D_{0}(\tau)+\varepsilon D_{1}(\tau)+\varepsilon^{2} D_{2}(\tau)+\cdots
\end{aligned}
$$

which can be truncated to exponentially small order, just as the adiabatic solution (6). In particular, $S_{0}(\tau)$ is the matrix diagonalizing $A$ statically, and the entries of $D_{0}(\tau)$ are eigenvalues of $A(\tau)$. The proof uses the fact that $a_{1}(\tau) \neq a_{2}(\tau)$.

In the specific case of the Lorenz equations, a linear transformation given to leading order by

$$
x_{1}=\sigma\left(y+z_{1}\right)+\mathcal{O}(\varepsilon), \quad x_{2}=\frac{\sigma-1}{2}\left(y+z_{1}\right)+s\left(y-z_{1}\right)+\mathcal{O}(\varepsilon), \quad x_{3}=z_{2}
$$

yields the equation

$$
\begin{aligned}
& \varepsilon \dot{y}=d_{1}(\tau) y+b_{1}(y, z, \tau) \\
& \varepsilon \dot{z}=D_{2}(\tau) z+b_{2}(y, z, \tau),
\end{aligned}
$$

where $d_{1}(\tau)=a_{1}(\tau)+\mathcal{O}(\varepsilon), D_{2}(\tau)$ is diagonal with entries $a_{2}(\tau)+\mathcal{O}(\varepsilon)$ and $a_{3}$, and $b_{1}, b_{2}$ are quadratic.

In order to deal with the nonlinear terms, we introduce invariant manifolds. Consider the partial differential equation

$$
\varepsilon \partial_{\tau} v(y, \tau)=D_{2}(\tau) v(y, \tau)+b_{2}(y, v, \tau)-\partial_{y} v(y, \tau)\left[d_{1}(\tau) y+b_{1}(y, v, \tau)\right] .
$$

It can be shown that this equation admits, in a neighborhood of $y=0$, a solution $v(y, \tau)=\mathcal{O}\left(y^{2}\right)$. When $r>1$, it lies at a distance of order $\varepsilon$ from the instantaneous unstable manifold of the origin, but it can be continued to times where $r<1$. The change of variables $z=\zeta+v(y, \tau)$ transforms the second equation of (24) into

$$
\varepsilon \dot{\zeta}=\left[D_{2}(\tau)+\beta_{2}(y, \zeta, \tau)\right] \zeta,
$$

where $\beta_{2}$ is of order $|y|+\|\zeta\|$. This equation admits $\zeta=0$ as invariant manifold. A similar change of variables $y=\eta+u(\zeta, \tau)$ transforms the first equation into

$$
\varepsilon \dot{\eta}=\left[d_{1}(\tau)+\beta_{1}(\eta, \zeta, \tau)\right] \eta
$$

\footnotetext{
${ }^{1}$ These matrices are not unique, since every column of $S$ can be multiplied by a function of time, which will of course affect terms of order $\varepsilon$ in $D$.
} 


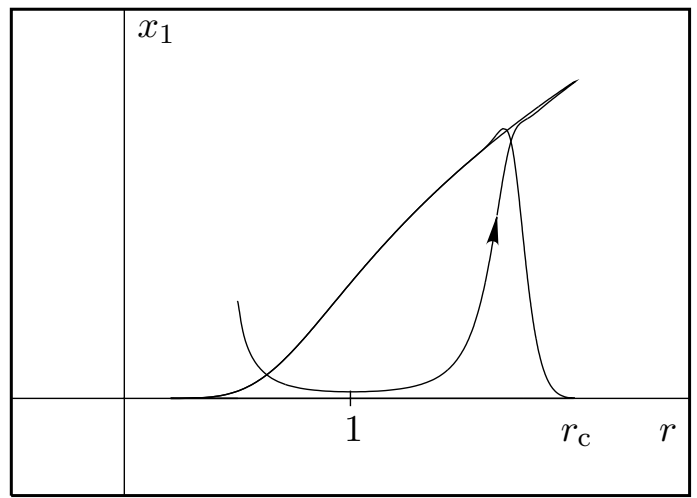

FiguRE 4. Rotation frequency of convection rolls $x_{1}$ as a function of the periodically varying temperature difference $r$ in the Lorenz model. After a first transient cycle, the motion settles on a hysteresis cycle, on which $x_{1}$ increases rapidly and decreases slowly.

defining a stable manifold separating the basins of attraction of $C_{+}$and $C_{-}$.

Since $D_{2}$ has negative eigenvalues, one easily shows that $\zeta(\tau)$ goes to zero exponentially fast. Thus the effective dynamics will take place on the invariant manifold $z=v(y, \tau)$, where it is governed by the scalar equation

$$
\begin{aligned}
\varepsilon \dot{y} & =d_{1}(\tau) y+h(\tau) y^{3}+\mathcal{O}\left(y^{5}\right), \\
h(\tau) & =-\frac{\sigma^{2}}{2 b s}\left(\frac{\sigma-1}{2}+s\right)+\mathcal{O}(\varepsilon) .
\end{aligned}
$$

$(y=\eta$ on the unstable manifold $\zeta=0)$. As $r(\tau)$ is varied periodically around $r=1$, $d_{1}(\tau)=a_{1}(\tau)+\mathcal{O}(\varepsilon)$ changes sign. The situation is thus very similar to the last example of Section 2. Assume that $d_{1}(\tau)$ becomes negative at $\tau_{0}$, positive at $\tau_{1}$, and negative again at $\tau_{0}+1$. Asymptotically, the solution will stay close to the origin for $\tau_{0}+n \leqslant \tau \leqslant \hat{\tau}+n$, where $\hat{\tau}>\tau_{1}$ is the delay time defined by the relation

$$
\int_{\tau_{0}}^{\hat{\tau}} d_{1}(\tau) \mathrm{d} \tau=0
$$

(this time exists if the average of $d_{1}(\tau)$ over one period is positive, otherwise solutions stay indefinitely close to the origin). For $\hat{\tau}+n<\tau<\tau_{0}+n+1$, the asymptotic solution follows $C_{+}$or $C_{-}$adiabatically. Thus the bifurcation delay leads once again to hysteresis (Fig. 1).

When $r(\tau)$ is varied back and forth (at least when $r$ does not become too large), the solution always follows the same equilibrium (which one it chooses depends on the initial condition). In the case of Rayleigh-Bénard convection, $x_{1}$ measures the rotation frequency of convection rolls. When $r(\tau)$ is increased, these rolls will appear suddenly, with a positive frequency, at some $r_{\mathrm{c}}=r(\hat{\tau})>1$. When $r(\tau)$ is decreased again, they slowly decelerate to disappear smoothly as $r$ becomes smaller than 1. The rolls will always turn in the same direction. We believe that it would be interesting to try to observe this delay experimentally. 


\section{Hysteresis in mean field ferromagnets}

Hysteresis in ferromagnets has been known and studied experimentally for a long time. Interest in a microscopic understanding of hysteresis and associated scaling laws has been renewed by the numerical study of [RKP]. The internal dynamics of ferromagnets, however, is so complicated that its modeling by ordinary differential equations is not obvious. We will consider here a simple Curie-Weiss model, which can be described by an effective mean field equation.

Consider the Hamiltonian

$$
H(\sigma)=-\frac{1}{2 N} \sum_{i \neq j \in \Lambda}\left\langle\sigma_{i} \mid J \sigma_{j}\right\rangle-\sum_{i \in \Lambda}\left\langle h \mid \sigma_{i}\right\rangle,
$$

where $\Lambda$ is a subset of $\mathbb{Z}^{d}$ with $N$ sites, the spins $\sigma_{i}$ are unit vectors in $\mathbb{R}^{n}, J$ is a fixed coupling matrix and $h$ the magnetic field. We introduce a stochastic spin flip dynamics of Glauber type. The detailed balance condition [Ka] is satisfied by a transition probability by unit time of the form

$$
\begin{aligned}
w\left(\sigma^{\prime} \mid \sigma\right) & =\sum_{i \in \Lambda} \prod_{j \neq i} \delta\left(\sigma_{j}^{\prime}-\sigma_{j}\right) \mathrm{e}^{\beta\left\langle\sigma_{i}^{\prime} \mid h_{i}(\sigma)\right\rangle} g\left(h_{i}(\sigma)\right), \\
h_{i}(\sigma) & :=h+\frac{1}{N} J \sum_{j \neq i} \sigma_{j}
\end{aligned}
$$

where $\beta=T^{-1}$ is the inverse temperature, and $g(h)$ an arbitrary function.

To derive a deterministic equation of motion, we consider a sequence of systems with $N$ sites, $N \rightarrow \infty$. Under appropriate assumptions on the initial probability distribution, one can derive in the thermodynamic limit a deterministic equation for the magnetization $m$ of the form?

$$
\frac{\mathrm{d} m}{\mathrm{~d} t}=-m+\beta(J m+h) F_{n}(\beta\|J m+h\|),
$$

where $F_{n}(x)$ depends on the dimension $n$ of the spins. In particular,

$$
F_{1}(x)=\frac{\operatorname{th} x}{x}, \quad F_{2}(x)=\frac{1}{2}-\frac{1}{16} x^{2}+\mathcal{O}\left(x^{4}\right) .
$$

It can be shown that corrections to $m(t)$ resulting from a finite $N$ are of order $N^{-1 / 2}$, and obey a Langevin equation [Ma1, Ma2].

We will now describe some interesting properties of the 1D and 2D models in a slowly oscillating magnetic field, paying attention in particular to the mechanism of magnetization reversal and its influence on the shape of hysteresis cycles. 
a

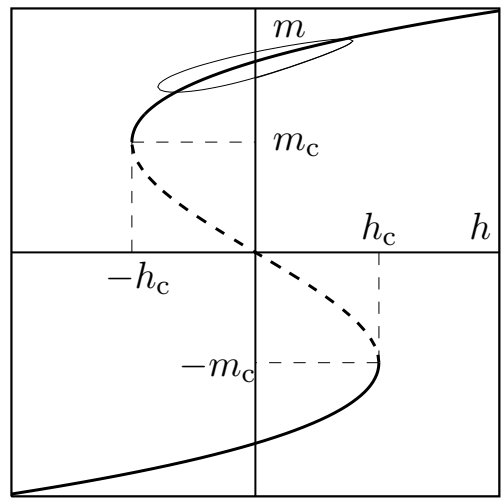

$\mathrm{b}$

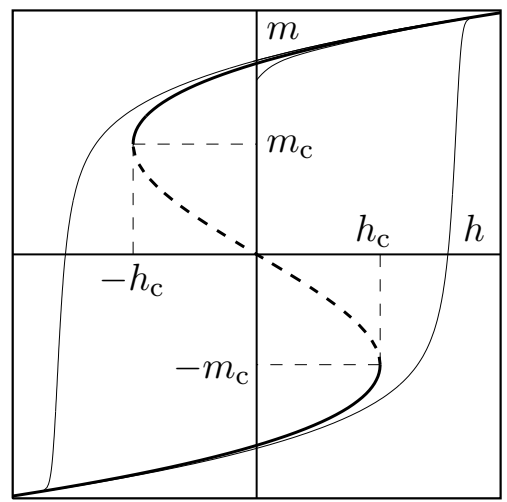

Figure 5. Solutions of (34) illustrating the phenomenon of "dynamic phase transition". When the amplitude $h_{0}$ of the magnetic field is smaller than the critical field $h_{\mathrm{c}}$, the magnetization oscillates around a nonzero average, and encloses an area of order $\varepsilon h_{0}$ (a). When $h_{0}$ is larger than $h_{\mathrm{c}}$, the average magnetization is zero, and the periodic solution encloses an area $\mathcal{A}(\varepsilon)=\mathcal{A}(0)+\mathcal{O}\left(\varepsilon^{2 / 3}\right)(\mathrm{b})$.

\subsection{One-dimensional case: dynamic phase transition}

For 1D spins, the adiabatic equation of motion can be written as

$$
\varepsilon \dot{m}=-m+\operatorname{th} \beta(J m+h(\tau)),
$$

where we will consider a periodic magnetic field of the form

$$
h(\tau)=h_{0} \sin (2 \pi \tau) .
$$

For positive inverse temperature $\beta=T^{-1}$, we may rescale the variables in such a way that $J=1$. If $\beta<1$, there is no static hysteresis. If $\beta>1$, the static bifurcation diagram is similar to the equation $\varepsilon \dot{x}=-x+x^{3}+\lambda(\tau)$ discussed in Section 22: it contains two saddle-node bifurcations $\left( \pm h_{\mathrm{c}}, \mp m_{\mathrm{c}}\right)$, where

$$
m_{\mathrm{c}}(T)=\sqrt{1-T}, \quad h_{\mathrm{c}}(T)=m_{\mathrm{c}}-T \operatorname{Argth} m_{\mathrm{c}} .
$$

As we have seen in Section 2, when $h_{0} \gg h_{\mathrm{c}}$ orbits are attracted by a hysteresis cycle with zero average magnetization (Fig. 5b) and area $\mathcal{A}(0)+\mathcal{O}\left(\varepsilon^{2 / 3}\right)$. This scaling law was already obtained in JGRM. When $h_{0}<h_{\mathrm{c}}$, the magnetization never sees any bifurcation point, and it follows asymptotically a cycle of width $\mathcal{O}(\varepsilon)$ with nonzero average magnetization (Fig. 5a).

This phenomenon was observed numerically and called "dynamic phase transition" by [TO], who also called "ferromagnetic" or $\mathbf{F}$-region the domain of $\left(T, h_{0}\right)$ plane where the asymptotic cycle has a nonzero magnetization, and "paramagnetic" or $\mathbf{P}$-region the domain where it has zero average magnetization. In the adiabatic limit, these regions are delimited by the line $h=h_{\mathrm{c}}(T)$. For positive $\varepsilon$, there

\footnotetext{
${ }^{2}$ This is the simplest equation, obtained for a particular choice of $g$. Other choices yield a multiplicative factor in the right-hand-side.

${ }^{3}$ We neglect here terms of order $\varepsilon$ stemming from the slow time dependence of $h$ in the derivation of the equation of motion.
} 
a

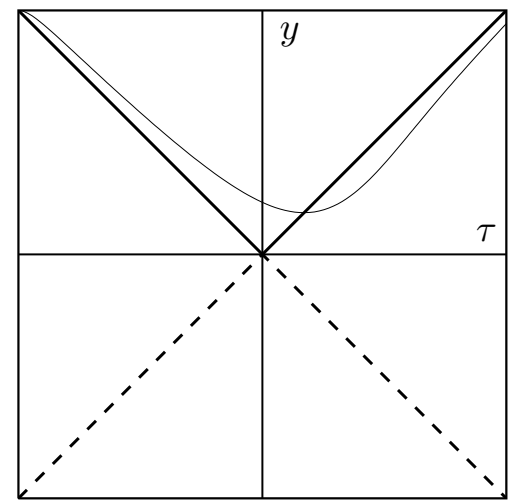

$\mathrm{b}$

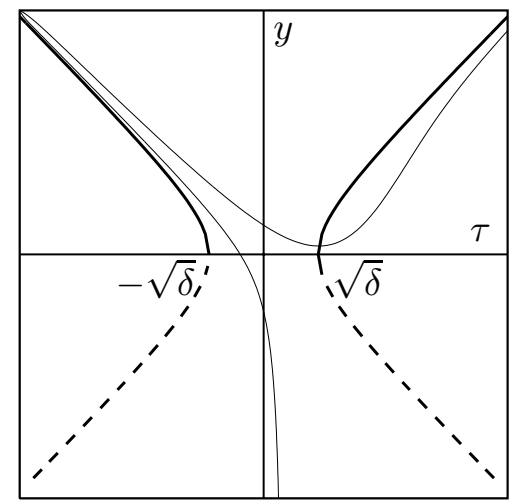

Figure 6. If the amplitude of the magnetic field is equal to $h_{0}=h_{\mathrm{c}}+\delta$, the motion near the turning point is governed by the Ricatti equation (38). If $\delta=0$, it describes a transcritical bifurcation, and adiabatic solutions follow the upper branch (a). This means that we are still in the F-region. For positive $\delta$, we show that this behaviour subsists as long as $\delta=\mathcal{O}(\varepsilon)$. In (b), trajectories are shown for two different values of $\varepsilon$. If $\varepsilon<\delta$, the solution escapes from below after a delay of order $\varepsilon^{2 / 3} \delta^{-1 / 6}$, and we have reached the $\mathrm{P}$-region.

may be a small overlap between these regions, where a symmetric $\mathrm{P}$-cycle and an asymmetric $\mathrm{F}$-cycle coexist.

We claim that for small $\varepsilon$ and $T=\beta^{-1}<1$, the $\mathrm{F}$-region grows by a distance of order $\varepsilon$, the $\mathrm{P}$-region shrinks by an amount of the same order (but may overlap the $\mathrm{F}$-region), and the corresponding cycles obey the scaling laws

$$
\begin{array}{ll}
\text { F-cycle: } & \mathcal{A}\left(\varepsilon, h_{0}\right) \approx h_{0} \varepsilon \text { if } h_{0}<h_{\mathrm{c}}, \quad \varepsilon \ln |\varepsilon| \text { if } h_{0}=h_{\mathrm{c}}, \\
\text { P-cycle: } & \mathcal{A}\left(\varepsilon, h_{0}\right) \approx \mathcal{A}_{0}+\varepsilon^{2 / 3}\left(h_{0}-h_{\mathrm{c}}\right)^{1 / 3} .
\end{array}
$$

Let us indicate how we obtain these scaling laws. Assume that $h_{0}=h_{\mathrm{c}}+\delta$. After translating the coordinates to the bifurcation point $\left(-h_{\mathrm{c}}, m_{\mathrm{c}}\right)$ and scaling them in a proper way, equation (34) becomes

$$
\varepsilon \dot{y}=-y^{2}-\delta+\tau^{2}+\text { higher order terms. }
$$

For $\delta=0$, this equation displays a transcritical bifurcation at the origin. For positive $\delta$, it splits up into two saddle-node bifurcations, with a gap of width $2 \sqrt{\delta}$ (Fig. (6). If $\delta<\mathcal{O}(\varepsilon)$, the transformation $\tau=\sqrt{\delta} \sigma, y=\sqrt{\varepsilon} z$ yields the equation

$$
\frac{\mathrm{d} z}{\mathrm{~d} \sigma}=-\tilde{\varepsilon} z^{2}+\tilde{\varepsilon}^{3}\left(\sigma^{2}-1\right), \quad \tilde{\varepsilon}=\sqrt{\delta / \varepsilon},
$$

which can be used to show that $z$ cannot move enough to slip through the gap, so that we are in the $\mathrm{P}$-region.

If $\delta>\mathcal{O}(\varepsilon)$, the transformation $\tau=\sqrt{\delta}(\sigma-1), y=\sqrt{\delta} z$ gives

$$
\left(\varepsilon \delta^{-1}\right) \frac{\mathrm{d} z}{\mathrm{~d} \sigma}=-z^{2}-\sigma+\sigma^{2}
$$



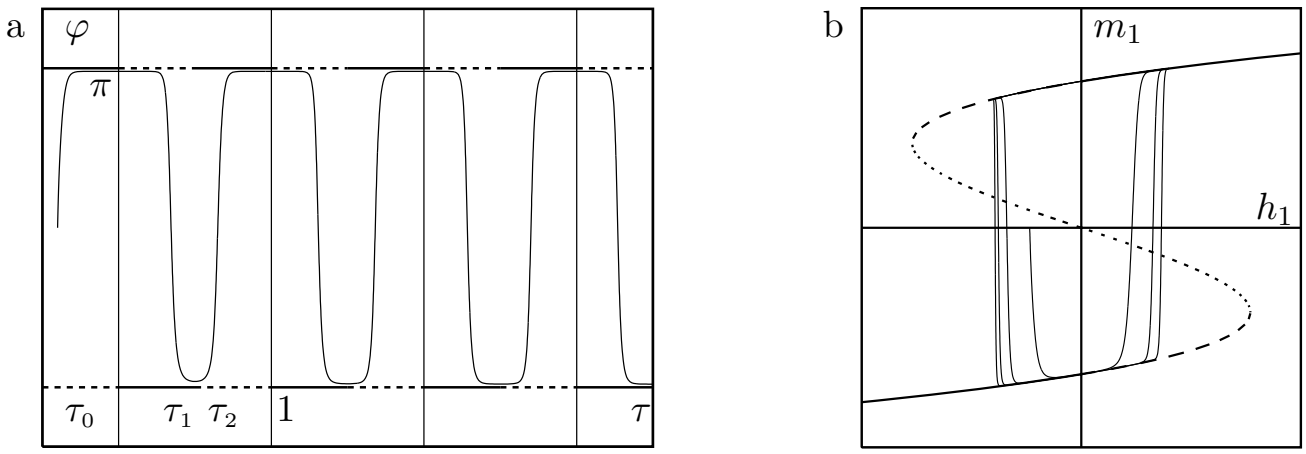

Figure 7. (a) Evolution of $\varphi(\tau)$, solution of equation (43). The magnetization quickly rotates at times $\tau_{n}$, determined recursively by the relation $\tau_{n+1}=\frac{n}{2}+$ $\Psi\left(\tau_{n}-\frac{n}{2}\right)$. (b) The plot of $m_{1}$ as a function of $h_{1}$ shows the asymptotic hysteresis cycle, which is determined solely by the delay times. Thick solid lines, dashed and dotted lines represent respectively sinks, saddles and sources of the static system. Due to bifurcation delay, the magnetization follows the hyperbolic branch for some time, but ultimately rotates around the unstable origin.

which is exactly the equation studied in Section 2. In particular, the trajectory slips through the gap after a time delay of order $\sqrt{\delta}\left(\varepsilon \delta^{-1}\right)^{2 / 3}=\varepsilon^{2 / 3} \delta^{-1 / 6}$. During this time, the magnetic field has reached a value $h_{0}+\mathcal{O}\left(\varepsilon^{2 / 3} \delta^{1 / 3}\right)$, which implies the scaling relation (37).

Finally, when $\delta \approx \varepsilon$, the trajectory may behave in either way. A more careful analysis of the Poincaré map shows that even though there is a small region where stable $\mathrm{F}$ - and $\mathrm{P}$-cycles can coexist, the transition is sharp, in the sense that the average magnetization jumps discontinuously from one cycle to another. In [TO], a smooth transition, where the magnetization goes to 0 continuously, has been observed for larger values of $\varepsilon$.

\subsection{Two-dimensional case: effect of anisotropy}

If we retain only the leading terms in (32), we obtain the Ginzburg-Landau equation

$$
\varepsilon \dot{m}=(\beta J-\mathbb{1}) m-\frac{1}{2} \beta J m\|\beta J m\|^{2}+\beta h,
$$

which describes the linearly driven, overdamped motion of a particle in a sombreroshaped potential. We shall assume that $h$ is parallel to an eigenvector of the symmetric matrix $J$. In the subspace of this eigenvector, the equation reduces to the previously studied case, and a minimal field amplitude $h_{\mathrm{c}}$ is necessary to reverse magnetization. When the magnetization has a transverse component, however, it can also turn around the potential maximum, for a much smaller field amplitude. We are thus going to focus on this situation.

In the isotropic case, we may choose $J=\mathbb{1}$ to obtain the equation

$$
\varepsilon \dot{m}=(\beta-1) m-\frac{1}{2} \beta^{3} m\|m\|^{2}+\beta h(\tau),
$$




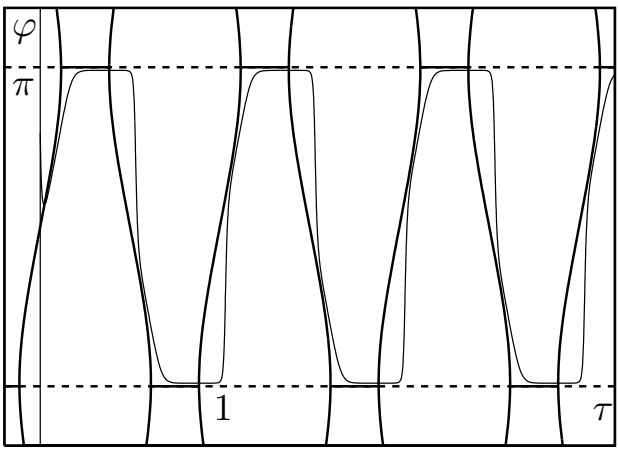

$\mathrm{b}$

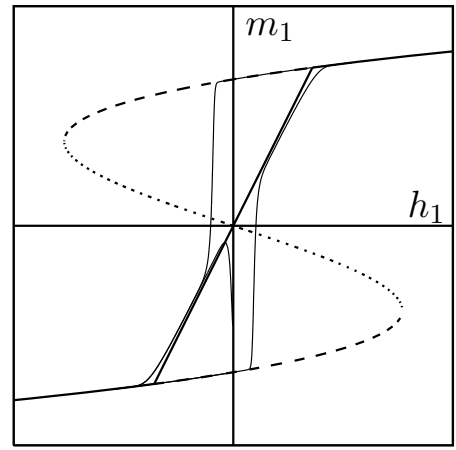

Figure 8. (a) Evolution of $\varphi$ in the anisotropic case with $\gamma>1$. Due to bifurcation delay, $\varphi$ spends some time near 0 or $\pi$, even when these points are unstable. It always drops back, however, to the transverse stable position. (b) The resulting hysteresis loop looks triangular. Curved lines are longitudinal equilibria, and the straight line represents transverse branches.

where we take a magnetic field $h(\tau)=\left(h_{1}(\tau), 0\right)$, with $h_{1}(\tau)=h_{0} \sin (2 \pi \tau)$. It is useful to write this equation in polar coordinates, with $m=(r \cos \varphi, r \sin \varphi)$, to get

$$
\begin{aligned}
\varepsilon \dot{r} & =(\beta-1) r-\frac{1}{2} \beta^{3} r^{3}+\beta h_{1}(\tau) \cos \varphi \\
\varepsilon \dot{\varphi} & =-\frac{\beta}{r} h_{1}(\tau) \sin \varphi .
\end{aligned}
$$

If $h_{1}\left(\tau_{0}\right)<0$, the magnetization settles near the left equilibrium, determined by $\varphi=\pi$ and $r=r_{-}\left(\tau_{0}\right)$, the largest solution of $(\beta-1) r-\frac{1}{2} \beta^{3} r^{3}-\beta h_{1}\left(\tau_{0}\right)=0$. When the field becomes positive, the phenomenon of bifurcation delay causes $\varphi$ to remain for some time in unstable equilibrium near $\pi$, until it switches to 0 at a time $\tau_{1}=\Psi\left(\tau_{0}\right)$, defined by

$$
\int_{\tau_{0}}^{\Psi\left(\tau_{0}\right)} \frac{h(\tau)}{r_{-}(\tau)} \mathrm{d} \tau=0 .
$$

Because of the symmetry, the next time of delayed magnetization reversal is then given by $\tau_{2}=\frac{1}{2}+\Psi\left(\tau_{1}-\frac{1}{2}\right)$ (Fig. 17). Subsequent reversal times are determined by the recursive formula $\tau_{n}=\frac{n}{2}+\Psi\left(\tau_{n}-\frac{n}{2}\right)$. It turns out that this self-determined bifurcation delay finally settles at a fixed point of the map $\tau \mapsto \Psi(\tau)-\frac{1}{2}$.

We now turn to the anisotropic case where $J=\left(\begin{array}{ll}1 & 0 \\ 0 & \gamma\end{array}\right)$. In the coordinates $m=\left(r \cos \varphi, \gamma^{-1} r \sin \varphi\right)$, the second equation of (43) becomes

$$
\varepsilon \dot{\varphi}=\beta\left(1-\frac{1}{2} \beta^{2} r^{2}\right)(\gamma-1) \sin \varphi \cos \varphi-\frac{\beta}{r} h_{1}(\tau) \sin \varphi .
$$

The case $\gamma<1$ is not very interesting, since the anisotropy enhances the effect of the magnetic field, and tends to align the magnetization with it. If $\gamma>1$, a new stable transversal equilibrium exists for small magnetic field. Its coordinates are determined by the relations $\|\beta J m\|^{2}=2\left(1-\beta^{-1} \gamma^{-1}\right)$ and $\left(1-\gamma^{-1}\right) m_{1}=\beta h_{1}$. The resulting hysteresis cycle is composed of two triangular loops (Fig. 8), since after 


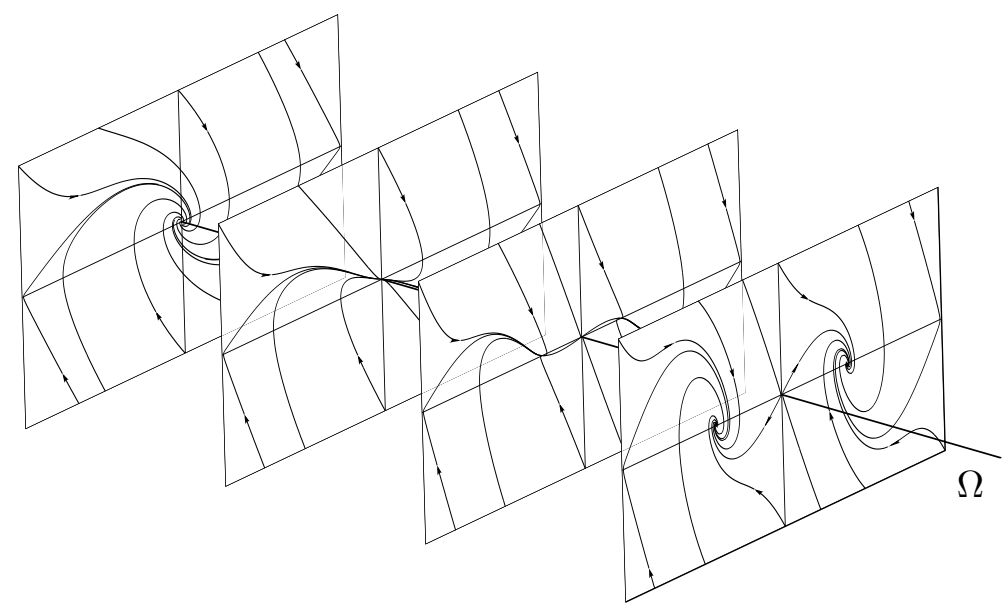

FiguRE 9. Phase portraits of the rotating pendulum for different values of the rotation frequency $\Omega$.

leaving the unstable position $\varphi=0$ or $\pi$, the magnetization drops to the transverse branch, which it follows until merging with the longitudinal branch.

We point out that if the magnetic field is slightly tilted with respect to the eigenvectors of $J$, the pitchfork bifurcations in Fig. 8 transform into saddle-nodes, which suppresses the bifurcation delay. The result is that instead of oscillating back and forth, the magnetization performs full circles, always in the same direction.

\section{Chaotic hysteresis of a rotating pendulum}

The examples we have considered up to now all described an overdamped, effectively one-dimensional motion, which displayed hysteretic, but not chaotic properties. We present here an example taking into account inertia, which turns out to have far more complicated dynamics.

Consider a mathematical pendulum mounted on a rotating table, turning with angular frequency $\Omega$. The pendulum is subject to weight, friction and a centrifugal torque, so that its equation of motion can be written in dimensionless variables

$$
\begin{aligned}
& \dot{q}=p \\
& \dot{p}=-2 \gamma p-\sin q+\Omega^{2} \sin q \cos q,
\end{aligned}
$$

where $q$ is the angle between pendulum and vertical, and $\gamma>0$ is a friction coefficient. This equation also describes the motion of a particle in a symmetric potential, shaped as a single well when $\Omega<1$ and as a double well when $\Omega>1$. The origin $O$ is always an equilibrium, while for $\Omega>1$, two new stable equilibria appear at

$$
Q_{ \pm}=\left( \pm q^{\star}(\Omega), 0\right), \quad q^{\star}(\Omega)=\operatorname{Arccos} \Omega^{-2} .
$$

The eigenvalues of the linearization of (46) around $O$ and $Q_{ \pm}$are given, respectively, by

$$
a_{ \pm}^{o}=-\gamma \pm \sqrt{\gamma^{2}+\Omega^{2}-1}, \quad a_{ \pm}^{\star}=-\gamma \pm \sqrt{\gamma^{2}-\Omega^{2}+\Omega^{-2}} .
$$



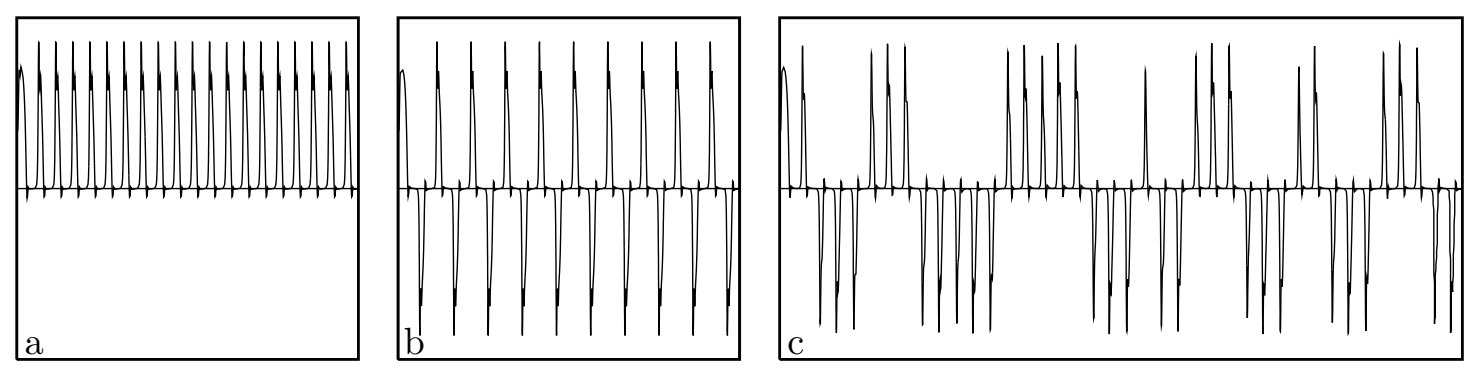

FiguRE 10. Solutions $q(\tau)$ for slightly different values of the adiabatic parameter $\varepsilon$. The time scale has been contracted in such a way as to show 20, resp. 40 periods of $\Omega$. One observes (a) solutions with the same period then $\Omega(\tau)$, (b) solutions with twice the period of $\Omega(\tau)$, going alternatively to one side and the other one, and (c), if $\varepsilon$ is carefully adjusted, solutions which have no apparent period.

There are four qualitatively different phase portraits, delimited by the values $\Omega=1$ and $\Omega=\Omega_{ \pm}(\gamma)$, where

$$
\Omega_{-}(\gamma)^{2}=1-\gamma^{2}, \quad \Omega_{+}(\gamma)^{2}=\frac{1}{2}\left[\gamma^{2}+\sqrt{\gamma^{2}+4}\right],
$$

namely (see Fig. 9):

- when $0<\Omega<\Omega_{-}(\gamma), O$ is a stable focus;

- when $\Omega_{-}(\gamma)<\Omega<1, O$ is a stable node;

- when $1<\Omega<\Omega_{+}(\gamma), O$ is a saddle and $Q_{ \pm}$are stable nodes;

- when $\Omega>\Omega_{+}(\gamma), O$ is a saddle and $Q_{ \pm}$are stable focuses.

If $\Omega=\Omega(\varepsilon t)$ is made slowly and periodically time-dependent, we obtain the adiabatic system

$$
\begin{aligned}
& \varepsilon \dot{q}=p \\
& \varepsilon \dot{p}=-2 \gamma p-\sin q+\Omega(\tau)^{2} \sin q \cos q .
\end{aligned}
$$

This system displays two interesting phenomena. The first one is a bifurcation delay similar to the one already observed in previous examples: when $\Omega$ is increased beyond 1 , the pendulum remains for some time in unstable equilibrium close to the origin, before joining one of the stable equilibria $Q_{+}$or $Q_{-}$. When $\Omega$ is decreased again below 1 , the pendulum follows this equilibrium until it joins the origin again, leading to hysteresis. The second interesting phenomenon is related to the sequence of visited equilibria, which depends on the value of the adiabatic parameter (Fig. 10). For some values, the pendulum always chooses the same equilibrium, just as the Lorenz system always chooses the same direction of rotation for the convection rolls. For other values of $\varepsilon$, however, one observes a sequence with twice the driving period, in which the pendulum visits alternatively the equilibria $Q_{+}$and $Q_{-}$. Between these periodic behaviours, it is even possible to observe apparently random sequences, which we called chaotic hysteresis [BK].

In order to explain this behaviour, we now compute an asymptotic expression for the Poincaré map in the $(q, p)$-plane, during one period of $\Omega(\tau)$. If $\Omega(\tau)$ remains within the interval $\left[\Omega_{-}, \Omega_{+}\right]$, the system can be reduced to $1 \mathrm{D}$ as in Section 3 , and 


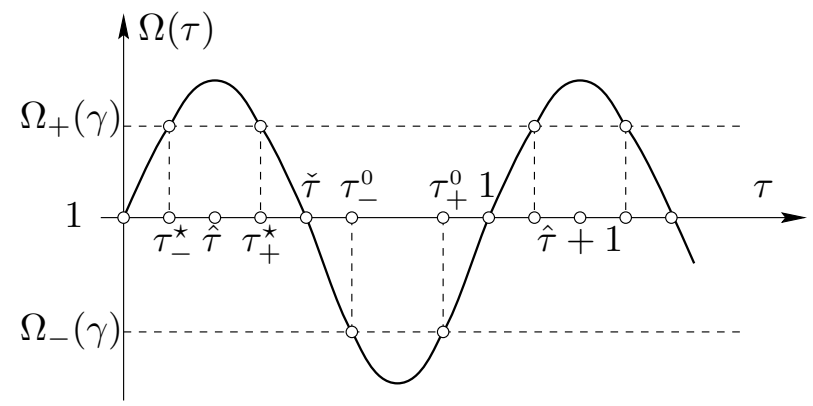

FiguRE 11. Function $\Omega(\tau)$ considered in the analysis. The instants when $\Omega$ crosses the values $\Omega_{ \pm}$and 1 delimit the different phases of the motion.

there is no possibility for chaotic motion. We thus consider the case where $\Omega(\tau)$ has a larger amplitude (Fig. 111). It is useful to introduce the notations

$$
\alpha^{o}\left(\tau_{2}, \tau_{1}\right)=\operatorname{Re} \int_{\tau_{1}}^{\tau_{2}} a_{+}^{o}(\tau) \mathrm{d} \tau, \quad \phi^{o}\left(\tau_{2}, \tau_{1}\right)=\operatorname{Im} \int_{\tau_{1}}^{\tau_{2}} a_{+}^{o}(\tau) \mathrm{d} \tau .
$$

Similar functions $\alpha^{\star}$ and $\phi^{\star}$ are defined for the linearizations around $Q_{ \pm}$.

For $\check{\tau}<\tau<1$, orbits are attracted by the stable origin. They remain close to it until a bifurcation delay time $\hat{\tau}+1$, defined by the relation $\alpha^{o}(\hat{\tau}+1, \check{\tau})=0$. During this part of motion, the system can be essentially described by its linearization around the origin. Except in a neighborhood of $\tau_{ \pm}^{o}$, where the eigenvalues $a_{ \pm}^{o}$ cross, we can carry out a dynamic diagonalization as in Section 3. The actual crossings are described by a local analysis, using Airy's equation. Combining these steps, we obtain that

$$
x(\hat{\tau}+1)=S(\hat{\tau}+1)\left(\begin{array}{cc}
\cos \left(\frac{\phi^{o}}{\varepsilon}\right) & \mathrm{e}^{-\delta_{2}^{o} / \varepsilon} \sin \left(\frac{\phi^{o}}{\varepsilon}+\theta_{2}^{o}\right) \\
-\mathrm{e}^{-\delta_{1}^{o} / \varepsilon} \sin \left(\frac{\phi^{o}}{\varepsilon}+\theta_{1}^{o}\right) & \mathrm{e}^{-\delta_{3}^{o} / \varepsilon} \cos \left(\frac{\phi^{o}}{\varepsilon}+\theta_{3}^{o}\right)
\end{array}\right) S(\check{\tau}) x(\check{\tau}),
$$

where $\phi^{o}=\phi^{o}\left(\tau_{+}^{0}, \tau_{-}^{0}\right)+\mathcal{O}(\varepsilon)$ is the dynamic phase of oscillations around the origin, and the columns of $S(\tau)$ are close to the eigenvectors associated with the origin. The positive factors $\delta_{j}^{o}$ describe the asymmetric contraction due to the difference between $a_{+}^{o}$ and $a_{-}^{o}$, and the $\theta_{j}^{o}$ are geometric phase shifts. It can be shown that the effect of nonlinear terms can be absorbed in these small geometric corrections.

The part of motion between $\hat{\tau}$ and $\check{\tau}$ is essentially nonlinear. Near the origin, we may use invariant manifolds as in Section 03 to transform (50) into

$$
\begin{aligned}
& \varepsilon \dot{\xi}=\left[a_{+}^{o}(\tau, \varepsilon)+\beta_{+}(\xi, \eta, \tau, \varepsilon)\right] \xi \\
& \varepsilon \dot{\eta}=\left[a_{-}^{o}(\tau, \varepsilon)+\beta_{-}(\xi, \eta, \tau, \varepsilon)\right] \eta,
\end{aligned}
$$

where $a_{ \pm}^{o}(\tau, \varepsilon)=a_{ \pm}^{o}(\tau)+\mathcal{O}(\varepsilon)$ and $\beta_{ \pm}$are of order $|\xi|+|\eta|$. Starting at $\hat{\tau}$ with a small initial condition $\left(\xi_{0}>0, \eta_{0}\right)$, the second equation in (53) shows that $\eta$ becomes exponentially small. The first one is used to prove that $\xi$ reaches a distance $d$ from the origin ( $d$ not too large) at a time $\bar{\tau}\left(\xi_{0}\right)+\mathcal{O}(\varepsilon)$, where

$$
\alpha^{o}\left(\bar{\tau}\left(\xi_{0}\right), \hat{\tau}\right)=-\varepsilon \ln \left(\xi_{0} / d\right)
$$



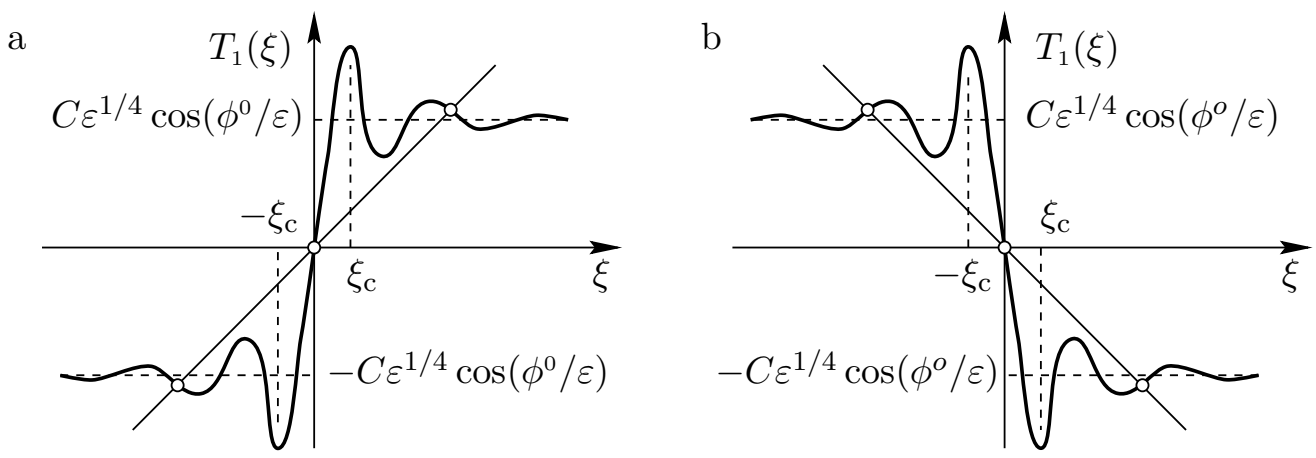

FiguRE 12. Schematic shape of the function $T_{1}(\xi)$ of equation (56) (a) for $\cos \left(\phi^{0} / \varepsilon\right)>0$ and $(\mathrm{b})$ for $\cos \left(\phi^{0} / \varepsilon\right)<0$. In the first case, there are two symmetric stable fixed points. In the second case, there is a stable orbit of period 2 .

provided $\xi_{0}>\xi_{\mathrm{c}}=\mathrm{e}^{-\alpha^{o}\left(\tau_{+}^{\star}, \hat{\tau}\right) / \varepsilon}$ (for smaller $\xi_{0}$, the orbit does not reach $Q_{+}$before the time $\left.\tau_{+}^{\star}\right)$. For $\tau>\bar{\tau}\left(\xi_{0}\right)$, the trajectory is attracted by $Q_{+}$, around which we carry out a similar analysis than around the origin, with the result

$$
\begin{aligned}
& \xi(\check{\tau})=C \varepsilon^{1 / 4}+\mathrm{e}^{\alpha^{\star} / \varepsilon} \cos \left(\frac{\phi^{\star}}{\varepsilon}\right) \\
& \eta(\check{\tau})=\mathrm{e}^{\left(\alpha^{\star}-\delta^{\star}\right) / \varepsilon} \sin \left(\frac{\phi^{\star}}{\varepsilon}+\theta^{\star}\right),
\end{aligned}
$$

where $\alpha^{\star}=\alpha^{\star}\left(\check{\tau}, \bar{\tau}\left(\xi_{0}\right)\right)+\mathcal{O}(\sqrt{\varepsilon})$ and $\phi^{\star}=\phi^{\star}\left(\tau_{+}^{\star}, \bar{\tau}\left(\xi_{0}\right)\right)+\mathcal{O}(\sqrt{\varepsilon})$, while $C, \delta^{\star}$ and $\theta^{\star}$ are constant at lowest order in $\varepsilon$. The position at $\check{\tau}$ thus depends essentially on $\xi_{0}$ through the delayed bifurcation time $\bar{\tau}\left(\xi_{0}\right)$, in such a way that (55) is the parametric equation of a squeezed spiral (which is essentially the image of the unstable manifold of the origin under the flow from $\hat{\tau}$ to $\check{\tau})$.

Combining this result with (52), we finally obtain a Poincaré map of the form

$$
\begin{aligned}
\xi_{1}=T_{1}\left(\xi_{0} ; \eta_{0}, \varepsilon\right)= & \cos \left(\frac{\phi^{o}}{\varepsilon}\right)\left[C \varepsilon^{1 / 4}+\mathrm{e}^{\alpha^{\star} / \varepsilon} \cos \left(\frac{\phi^{\star}}{\varepsilon}\right)\right] \\
& +\mathrm{e}^{\left(\alpha^{\star}-\delta\right) / \varepsilon} \sin \left(\frac{\phi^{o}}{\varepsilon}+\theta^{o}\right) \sin \left(\frac{\phi^{\star}}{\varepsilon}+\theta^{\star}\right), \\
\eta_{1}=T_{2}\left(\xi_{0} ; \eta_{0}, \varepsilon\right)= & \mathcal{O}\left(\mathrm{e}^{-\delta_{2}^{o}} / \varepsilon\right),
\end{aligned}
$$

where $\xi_{j}=\xi(\hat{\tau}+j)$ measures the distance to the stable manifold of the origin (it is close to $q)$, and $\eta_{j}=\eta(\hat{\tau}+j)$ measures the distance to the unstable manifold. This expression is valid for $\xi_{0}>0$, but is easily extended to negative $\xi_{0}$, since the Poincaré map is odd. The dynamics is thus essentially determined by the $1 \mathrm{D}$ map $\xi_{0} \mapsto T_{1}\left(\xi_{0} ; 0, \varepsilon\right)$, which is oscillating around $\pm C \varepsilon^{1 / 4} \cos \left(\frac{\phi^{o}}{\varepsilon}\right)$ (Fig. 12). One easily shows the existence of a positive constant $\mu$ such that, if $\varepsilon^{1 / 4} \cos \left(\phi^{o} / \varepsilon\right)>\mathrm{e}^{-\mu / \varepsilon}$, this map admits stable fixed points at $\pm \xi^{\star} \approx \pm \varepsilon^{1 / 4} \cos \left(\phi^{o} / \varepsilon\right)$, which correspond to cycles of period 1. When $\varepsilon^{1 / 4} \cos \left(\phi^{o} / \varepsilon\right)<-\mathrm{e}^{-\mu / \varepsilon}$, there is an orbit of period 2 , for which the pendulum alternatively visits the left and right equilibrium (Fig. 12). These properties can be shown to hold for the $2 \mathrm{D}$ map, which is confirmed by numerical simulations (Fig. 13).

Chaotic motion is possible in the intermediate regions, where $\left|\varepsilon^{1 / 4} \cos \left(\phi^{o} / \varepsilon\right)\right|<$ $\mathrm{e}^{-\mu / \varepsilon}$. For the $2 \mathrm{D}$ map, it is difficult to prove existence of such a motion, but one 

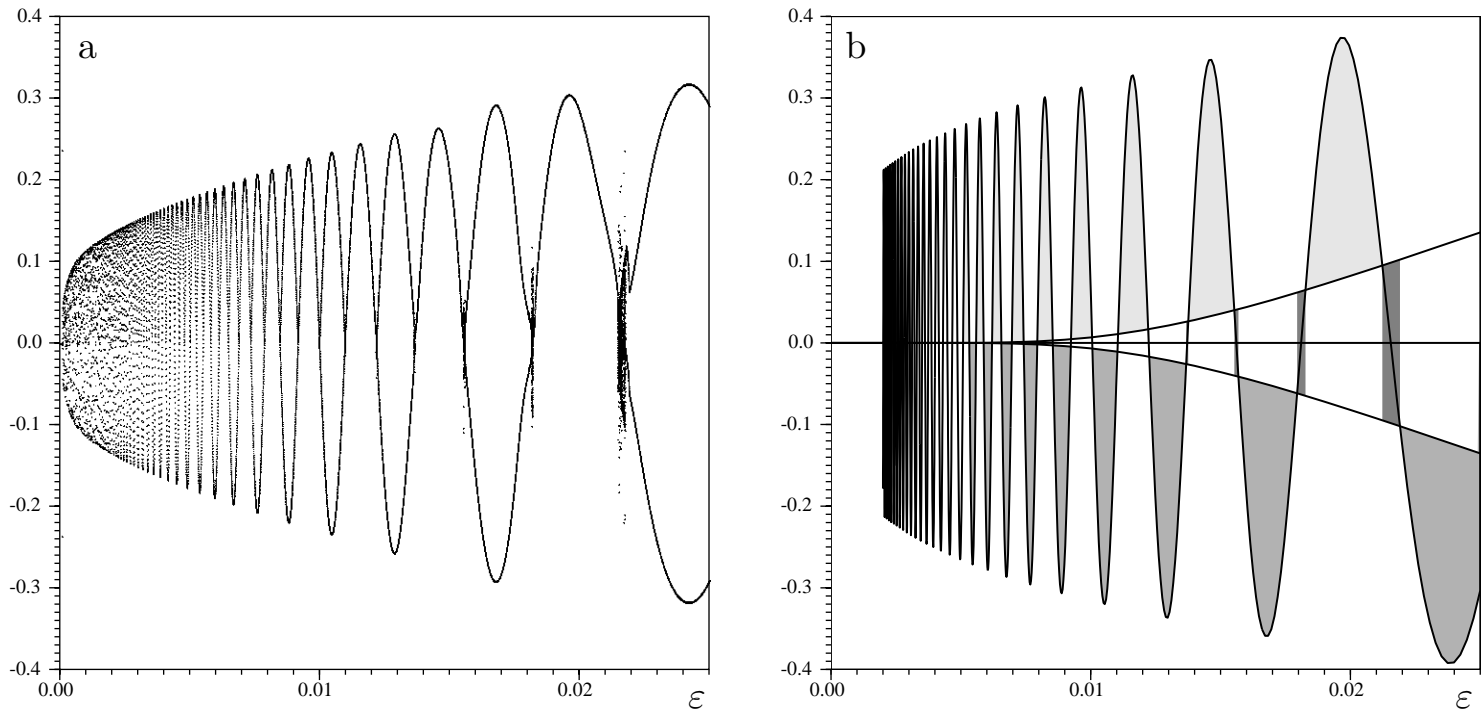

Figure 13. (a) Numerically computed bifurcation diagrams of the Poincaré map. For each value of $\varepsilon$, we have plotted the asymptotic value of $q(\hat{\tau}+n)$, for one initial condition. On the domain $0<\varepsilon<0.025$, the diagram clearly shows the alternates of regions with a period -1 and a period -2 cycle, separated by small chaotic zones. (b) Plots of the functions $\varepsilon^{1 / 4} \cos \left(\phi^{o} / \varepsilon\right)$ and $\pm \mathrm{e}^{-\mu / \varepsilon}$. Light gray zones are those where the theory predicts existence of a period-1 cycle, medium gray zones those with a period-2 cycle. Dark gray zone are those where chaotic hysteresis is possible, and, indeed, observed. We point out that in figure (b), the dynamic phase $\phi^{o}(0)$ has been computed analytically. Only the next-to-leadingorder correction to $\phi^{o}(\varepsilon)$ (which results in a phase shift) has been chosen in order to fit the numerical results.

$\mathrm{a}$

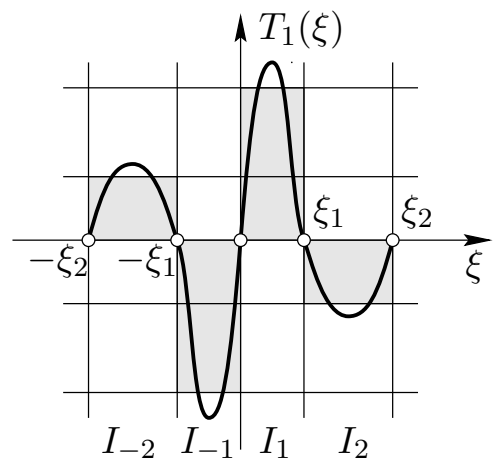

$\mathrm{b}$

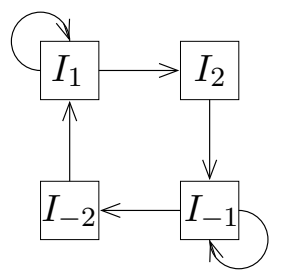

Figure 14. The interval map (a) admits the Markov subgraph (b), which allows for periodic orbits of all periods, except possibly 3 . 
can do more for the simplified 1D map, using symbolic dynamics. In fact, when $\cos \left(\phi^{o} / \varepsilon\right)=0$ and under certain conditions on $\Omega(\tau)$, one finds that $T_{1}(\xi)$ behaves as in Fig. 14a: it vanishes at two points $\xi_{1}$ and $\xi_{2}$, and, being odd, also at $-\xi_{1}$ and $-\xi_{2}$. These points define four intervals $I_{-2}, I_{-1}, I_{1}$ and $I_{2}$, and the maximum of $T_{1}$ on $I_{1}$ is larger than $\xi_{2}$, while its minimum on $I_{2}$ is smaller than $-\xi_{1}$. The Markov graph of $T_{1}$ is defined as the graph with sites $I_{j}$, admitting an oriented edge $I_{j} \rightarrow I_{k}$ whenever $T_{1}\left(I_{j}\right) \supset I_{k}$. It is known [MS that for every path in the Markov graph, there exists an orbit visiting the corresponding sequence of intervals. In particular, using Sarkovskii's theorem [MS], it is possible to prove the existence of periodic orbits of every period except possibly 3.

The mathematical pendulum analysed here has been realized experimentally, and all the phenomena predicted by the equations have been observed. They depend, in fact, only on a few qualitative features of the system. The origin should be a focus for some values of the parameter, in order to allow the orbits to wind around it. It should be hyperbolic for other values of the parameter, for which two new stable equilibria should exist. Chaotic motion requires, in addition, these asymmetric equilibria to be sometimes focuses, in order to create the oscillations in the Poincaré map. Under these conditions, it should be possible to observe chaotic hysteresis for other nonlinear oscillators (see Section 6.2).

\section{Examples of eigenvalue crossings}

We mentioned in Section 3 that adiabatic linear systems of the form $\varepsilon \dot{x}=A(\tau) x$ could be diagonalized (and thus solved) by the change of variables $x=S(\tau ; \varepsilon) y$, where $S$ is a matrix satisfying the equation

$$
\varepsilon \dot{S}=A S-S D
$$

and $D$ is a suitable diagonal matrix. This procedure is only useful, however, if we manage to control the transformation matrix $S$, which should be bounded (e.g. close to the matrix $S_{0}(\tau)$ which diagonalizes $A$ statically). Such a control turns out to be possible at least in two cases: when the eigenvalues of $A(\tau)$ have different real parts, or, (in a more restricted sense) when they have the same real part but different imaginary parts. I

This leaves open the question of the effect of different types of eigenvalue crossings. The most generic case, when $A$ is not diagonalizable at the crossing time, has been mentioned in Section 5. It can be studied using the properties of Airy functions (see also $\mathbf{W a}$ ). In the present section, we illustrate the effect of two other types of crossing. The first one occurs when $A(\tau)$ is symmetric, and can thus be diagonalized even when it has identical eigenvalues. The second one arises when the eigenvalues' real parts cross, but their imaginary parts are different. We call this situation eigenvalue cruising; it is closely related to properties of dynamic Hopf bifurcations discussed in Ne1, Ne2].

\footnotetext{
${ }^{4}$ More generally, the system can be bloc-diagonalized when the eigenvalues can be split into two groups with non-crossing real parts.
} 


\subsection{Symmetric crossing}

Let us consider the overdamped motion of a particle in the $2 \mathrm{D}$ potential

$$
\Phi(x, \varepsilon t)=-\frac{1}{2}\langle x \mid A(\varepsilon t) x\rangle+\frac{1}{4}\|x\|^{4},
$$

where $A$ is a symmetric matrix. The equation of motion can be written

$$
\varepsilon \dot{x}=A(\tau) x-\|x\|^{2} x
$$

We assume that the matrix $A(\tau)$ is given by

$$
A(\tau)=a(\tau)\left(\begin{array}{rr}
\cos 2 \theta(\tau) & \sin 2 \theta(\tau) \\
\sin 2 \theta(\tau) & -\cos 2 \theta(\tau)
\end{array}\right)
$$

so that it admits eigenvalues $\pm a(\tau)$ and eigenvectors $v_{1}=(\cos \theta, \sin \theta)$ and $v_{2}=$ $(-\sin \theta, \cos \theta)$. Thus, the potential $\Phi$ has minima at $\pm \sqrt{a} v_{1}$ if $a$ is positive, and at $\pm \sqrt{-a} v_{2}$ if $a$ is negative. To diagonalize the linearized equation $\varepsilon \dot{x}=A(\tau) x$, we may try to solve equation (57) with matrices $S$ and $D$ of the form

$$
S=\left(\begin{array}{rr}
\cos \theta_{1}(\tau) & -\sin \theta_{2}(\tau) \\
\sin \theta_{1}(\tau) & \cos \theta_{2}(\tau)
\end{array}\right), \quad D=\left(\begin{array}{cc}
d_{1}(\tau) & 0 \\
0 & d_{2}(\tau)
\end{array}\right)
$$

Substitution of this Ansatz in (57) yields the relations

$$
\begin{array}{ll}
\varepsilon \dot{\theta}_{1}=-a(\tau) \sin 2\left(\theta_{1}-\theta(\tau)\right), & d_{1}(\tau)=a(\tau) \cos 2\left(\theta_{1}-\theta(\tau)\right), \\
\varepsilon \dot{\theta}_{2}=a(\tau) \sin 2\left(\theta_{2}-\theta(\tau)\right), & d_{2}(\tau)=-a(\tau) \cos 2\left(\theta_{2}-\theta(\tau)\right) .
\end{array}
$$

If $a(\tau)$ does not vanish (i.e., when there is no eigenvalue crossing), these equations admit equilibrium branches at $\theta_{1}=\theta_{2}=\theta(\tau)$, of opposite stability. By the results of Section 2, we know that they admit particular adiabatic solutions $\theta_{1}(\tau)=\theta(\tau)+\mathcal{O}(\varepsilon)$ and $\theta_{2}(\tau)=\theta(\tau)+\mathcal{O}(\varepsilon)$. The evolution operator of the linearized system can thus be written

$$
\begin{gathered}
U\left(\tau, \tau_{0}\right)=S(\tau)\left(\begin{array}{cc}
\mathrm{e}^{\delta_{1}\left(\tau, \tau_{0}\right) / \varepsilon} & 0 \\
0 & \mathrm{e}^{\delta_{2}\left(\tau, \tau_{0}\right) / \varepsilon}
\end{array}\right) S\left(\tau_{0}\right)^{-1} \\
\delta_{1,2}\left(\tau, \tau_{0}\right)=\int_{\tau_{0}}^{\tau} d_{1,2}(s) \mathrm{d} s= \pm \int_{\tau_{0}}^{\tau} a(s) \mathrm{d} s+\mathcal{O}\left(\varepsilon^{2}\right) .
\end{gathered}
$$

The columns of $S(\tau)$ can be considered as dynamic eigenvectors which are close to the static eigenvectors $v_{1,2}$. They define invariant subspaces (depending on $\tau$ ), in which the motion is expanding, resp. contracting.

When $a(\tau)$ is allowed to vanish, new phenomena occur because the equations (62), (63) undergo bifurcation. It is instructive to consider the case $a(\tau)=-\cos \tau$, for three different functions $\theta(\tau):(1) \theta(\tau)=0$, (2) $\theta(\tau)=-\cos \tau$ and (3) $\theta(\tau)=\tau$.

If $\theta(\tau)=0$, (62) admits the solution $\theta_{1}=\theta_{2} \equiv 0$, and $d_{1,2}= \pm a(\tau)$. The evolution operator can thus be written

$$
U(\tau, 0)=\left(\begin{array}{cc}
\mathrm{e}^{-\sin (\tau) / \varepsilon} & 0 \\
0 & \mathrm{e}^{\sin (\tau) / \varepsilon}
\end{array}\right)
$$


a

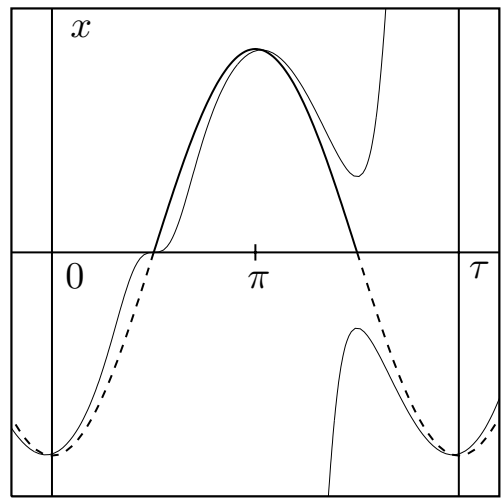

$\mathrm{b}$

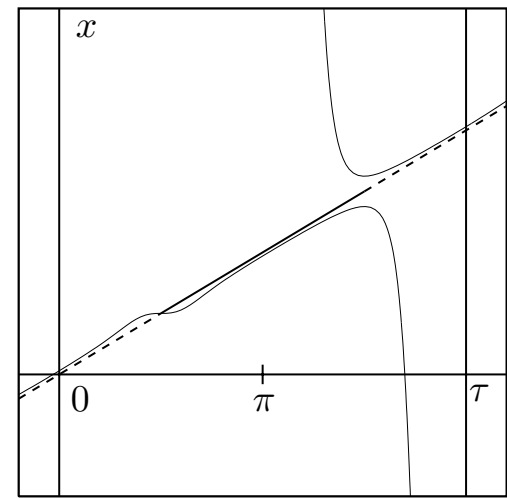

Figure 15. Solutions (thin lines) of (62) when $a(\tau)=-\cos \tau$ : (a) in the case $\theta(\tau)=-\cos \tau,(\mathrm{b})$ in the case $\theta(\tau)=\tau$. In both cases, one can construct a particular solution remaining close to the static equilibrium $\theta(\tau)$ (thick lines, where the solid lines indicate stable branches and the dashed lines unstable ones), admitting a discontinuity at $\tau=\frac{3 \pi}{2}$. Solutions of (63) behave in a similar way, with a discontinuity at $\tau=\frac{\pi}{2}$.

The subspaces $x_{1}=0$ and $x_{2}=0$ are invariant. If $x_{2}(0)=0, x_{1}(\tau)$ remains exponentially small until $\tau=\pi$, which is the standard bifurcation delay.

If $\theta(\tau)=-\cos \tau$, it is not possible to construct solutions of (62) remaining indefinitely close to $\theta(\tau)$. The best one can do is to construct periodic solutions $\theta_{1}(\tau)$ and $\theta_{2}(\tau)$ admitting a discontinuity of order $\sqrt{\varepsilon}$, respectively at times $\frac{3 \pi}{2}$ and $\frac{\pi}{2}$ (Fig. 15a). As a result, for $\frac{\pi}{2}<\tau<\frac{3 \pi}{2}$ we have

$$
U(\tau, 0)=S(\tau)\left(\begin{array}{cc}
\mathrm{e}^{\delta_{1}(\tau, \pi / 2) / \varepsilon} & 0 \\
0 & \mathrm{e}^{\delta_{2}(\tau, \pi / 2) / \varepsilon}
\end{array}\right) T\left(\begin{array}{cc}
\mathrm{e}^{\delta_{1}(\pi / 2,0) / \varepsilon} & 0 \\
0 & \mathrm{e}^{\delta_{2}(\pi / 2,0) / \varepsilon}
\end{array}\right) S(0)^{-1},
$$

where

$$
T=S\left(\frac{\pi}{2}+\right)^{-1} S\left(\frac{\pi}{2}-\right)=\left(\begin{array}{cc}
1+\mathcal{O}(\sqrt{\varepsilon}) & \sin \left(\theta_{2}^{+}-\theta_{2}^{-}\right)+\mathcal{O}(\varepsilon) \\
0 & 1
\end{array}\right)
$$

with $\theta_{2}^{ \pm}=\theta_{2}\left(\frac{\pi}{2} \pm\right)$. The off-diagonal term of this matrix induces a transition between the directions which were invariant before $\tau=\frac{\pi}{2}$. In particular, when $\tau=\pi$, we have to leading order in $\varepsilon$

$$
U(\pi, 0) \approx\left(\begin{array}{cc}
1 & \sin \left(\theta_{2}^{+}-\theta_{2}^{-}\right) \mathrm{e}^{2 / \varepsilon} \\
0 & 1
\end{array}\right) .
$$

This transformation rotates the vertical axis by almost $\pi / 2$. The matrix $U(2 \pi, \pi)$ is found to rotate the horizontal axis by almost $-\pi / 2$. This means that there exists no invariant subspace in which the particle performs an independent motion. The eigenvalue crossing thus results in an interaction between both modes, with the particle always following the most unstable direction. The sign of the discontinuities of $\theta_{1,2}$ is important: in this case it induces a back-and-forth oscillation of the particle between two wells (Fig. 16a). 
a

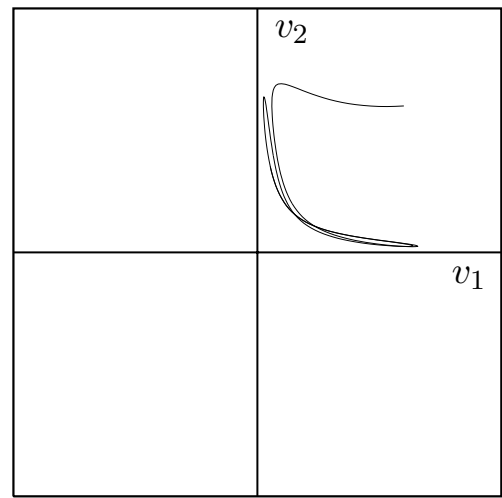

$\mathrm{b}$

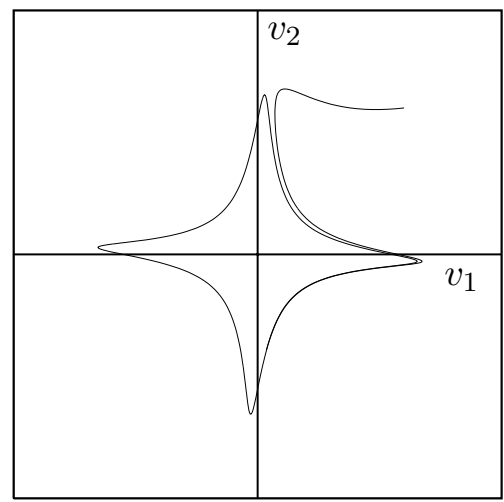

Figure 16. Solutions of (58) when $a(\tau)=-\cos \tau$, plotted with respect to the rotating reference frame $\left(v_{1}, v_{2}\right)$ : (a) in the case $\theta(\tau)=-\cos \tau$, the particle oscillates back and forth between two potential wells, while (b) in the case $\theta(\tau)=\tau$ it visits all four wells in a row.

In the case $\theta(\tau)=\tau$, the situation is similar, but with a discontinuity of $\theta_{1}$ of opposite sign (Fig. 15b). As a result, the coordinate axes are always rotated in the same direction, and the particle visits all four wells in a row (Fig. 16b).

\subsection{Coupled oscillators and eigenvalue cruising}

We call eigenvalue cruising the situation arising when some eigenvalues of the matrix $A$ drift past one another at some imaginary distance. This cruising also leads to an interaction between the modes; however, unlike in the case of diagonal crossing, this interaction is delayed.

Eigenvalue cruisings appear in particular in coupled oscillators. Consider for instance the system

$$
\begin{array}{r}
\ddot{q}_{1}+2 \gamma_{1} \dot{q}_{1}+\left(1-\lambda+q_{1}^{2}+4 q_{2}^{2}\right) q_{1}-\mu q_{2}=0, \\
\ddot{q}_{2}+2 \gamma_{2} \dot{q}_{2}+4\left(4-\lambda+q_{1}^{2}+4 q_{2}^{2}\right) q_{2}+\mu q_{1}=0,
\end{array}
$$

which was introduced by Kobayashi [Ko to describe the vibrations of a buckled plate with supersonic flow on one side of the plate. The variables $q_{1}$ and $q_{2}$ are amplitudes of the two dominant Fourier modes of the deflection, $\lambda$ is the in-plane compressive stress, $\mu$ the dynamic fluid pressure of the supersonic flow, and $\gamma_{1,2}$ are friction coefficients (which were taken equal in [Ko]).

Introducing $p_{1}=\dot{q}_{1}$ and $p_{2}=\dot{q}_{2}$, (69) can be written as a $4 \mathrm{D}$ first order system for the variables $\left(q_{1}, p_{1}, q_{2}, p_{2}\right)$, which admits the origin as an equilibrium. The linearization around the origin is a $4 \times 4$ matrix with eigenvalues

$$
\begin{aligned}
& a_{1, \pm}=-\gamma_{1} \pm \sqrt{\gamma_{1}^{2}+\lambda-1}+\mathcal{O}\left(\mu^{2}\right), \\
& a_{2, \pm}=-\gamma_{2} \pm \sqrt{\gamma_{2}^{2}+4(\lambda-4)}+\mathcal{O}\left(\mu^{2}\right) .
\end{aligned}
$$

An eigenvalue cruising arises for instance in the following situation: assume $\gamma_{1}=2$, $\gamma_{2}=1$ and $\mu=0$, so that $a_{1, \pm}=-2 \pm \sqrt{\lambda+3}$ and $a_{2, \pm}=-1 \pm \mathrm{i} \sqrt{15-4 \lambda}$. As 


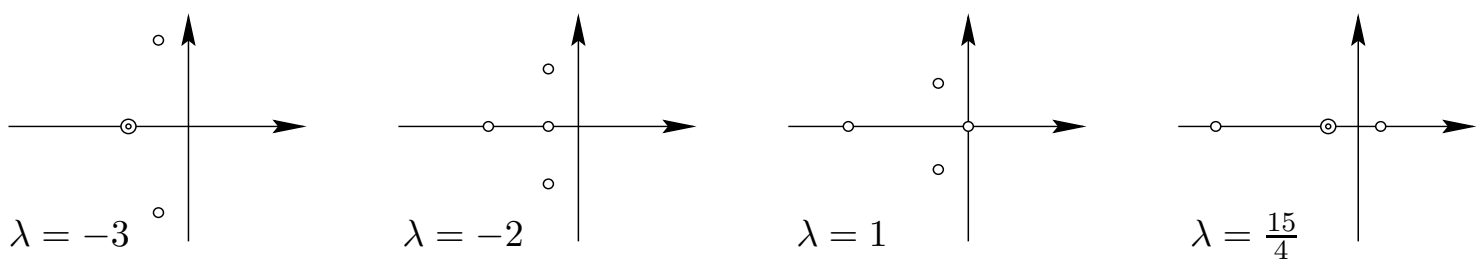

Figure 17. Eigenvalues of the linearized Kobayashi equations for $\gamma_{1}=2, \gamma_{2}=1$ and $\mu=0$. There is an eigenvalue cruising for $\lambda=-2$.

$\lambda$ increases from -3 to $\frac{15}{4}$, the complex eigenvalues $a_{2, \pm}$ correspond to oscillations, while the real eigenvalues $a_{1, \pm}$ describe an overdamped motion. There is a cruising at $\lambda=-2$ and the origin becomes unstable at $\lambda=1$ (Fig. 17). The same qualitative features hold for small positive coupling $\mu$.

We are interested in the following question. Assume that $\lambda$ is increased monotonically and adiabatically, starting with a generic initial condition at a time $\tau_{0}$ where $\lambda$ is smaller than 1 . For what value of $\lambda$ does the trajectory depart from the origin? The answer turns out to be related in a rather subtle way to bifurcation delay and eigenvalue cruising. It is easier to explain this phenomenon on the simple model equation

$$
\varepsilon \dot{x}=A(\tau) x, \quad A(\tau)=\left(\begin{array}{cc}
a_{1}(\tau) & \mu \\
-\mu & a_{2}(\tau)
\end{array}\right), \quad \begin{array}{ll}
a_{1}(\tau)=-1+\tau \\
a_{2}(\tau)=-1+\mathrm{i} .
\end{array}
$$

As in Kobayashi's equations, $a_{1}$ represents the overdamped mode, and $a_{2}$ the oscillating one (in complex notation). The cruising occurs at $\tau=0$. To diagonalize this equation, we try to solve the equation $\varepsilon \dot{S}=A S-S D$ with the Ansatz

$$
S(\tau)=\left(\begin{array}{cc}
1 & s_{2}(\tau) \\
s_{1}(\tau) & 1
\end{array}\right), \quad D(\tau)=\left(\begin{array}{cc}
d_{1}(\tau) & 0 \\
0 & d_{2}(\tau)
\end{array}\right) .
$$

Substitution in the equation for $S$ yields the relations

$$
\begin{array}{ll}
\varepsilon \dot{s}_{1}=-\mu-(\tau-\mathrm{i}) s_{1}-\mu s_{1}^{2}, & d_{1}=a_{1}+\mu s_{1}, \\
\varepsilon \dot{s}_{2}=\mu+(\tau-\mathrm{i}) s_{2}+\mu s_{2}^{2}, & d_{2}=a_{2}-\mu s_{2} .
\end{array}
$$

The first equation has a static equilibrium at $s_{1}^{\star}(\tau)=-\mu /(\tau-i)+\mathcal{O}\left(\mu^{2}\right)$, which is unstable for $\tau<0$ and stable for $\tau>0$. One can show that the solution of (73) with initial condition $s_{1}(0)=s_{1}^{\star}(0)$ tracks the branch $s_{1}^{\star}(\tau)$ at a distance at most $\mathcal{O}(\varepsilon)$, for both negative and positive times.

The second equation has a more subtle behaviour. It admits an equilibrium branch at $s_{2}^{\star}(\tau)=\mu /(\tau-\mathrm{i})+\mathcal{O}\left(\mu^{2}\right)$, which is stable for $\tau<0$ and unstable for $\tau>0$; in fact, it undergoes Hopf bifurcation. Such bifurcations have been studied by Neishtadt [Ne1, Ne2, Ne3]. The interesting fact is that there exist solutions tracking the equilibrium branch beyond the bifurcation point, but only until a time called maximal delay or buffer point. This point is obtained in the following way: let $a(\tau)=\tau-\mathrm{i}+\mathcal{O}\left(\mu^{2}\right)$ be the linearization of (74) around the equilibrium 
a

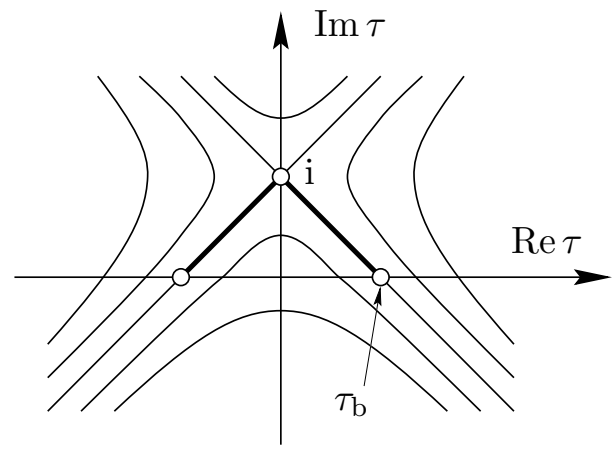

b

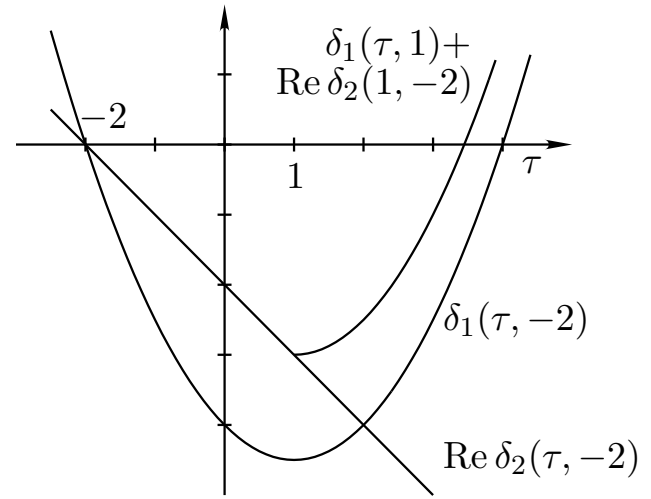

Figure 18. (a) The level lines of the function $\operatorname{Re} \alpha(\tau)$ of equation (75) are hyperbolas centered at $\tau=\mathrm{i}$. The largest positive time which can be connected to the negative real axis by such a line is the buffer time $\tau_{\mathrm{b}}=1$. (b) The origin becomes unstable when the largest exponent of a matrix element of the evolution operator (77) becomes positive. This may happen earlier when $\mu \neq 0$, because the oscillators interact at the buffer time.

$s_{2}^{\star}(\tau)$. Define the function

$$
\operatorname{Re} \alpha(\tau)=\operatorname{Re} \int_{0}^{\tau} a(s) \mathrm{d} s=\frac{1}{2}\left[(\operatorname{Re} \tau)^{2}-(\operatorname{Im} \tau-1)^{2}+1\right]+\mathcal{O}\left(\mu^{2}\right) .
$$

The buffer time $\tau_{\mathrm{b}}$ is the largest real time which can be connected to the negative real axis by a path of constant $\operatorname{Re} \alpha$ (and with some additional properties given in Ne3 ). In the present situation, $\tau_{\mathrm{b}}=1$ (Fig. 18a). As a consequence, for $\tau \leqslant 1$, we can construct a solution of (74) which is close to $s_{2}^{\star}(\tau)$, and the evolution operator of (71) is given by

$$
\begin{aligned}
& U\left(\tau, \tau_{0}\right)=S(\tau)\left(\begin{array}{cc}
\mathrm{e}^{\delta_{1}\left(\tau, \tau_{0}\right) / \varepsilon} & 0 \\
0 & \mathrm{e}^{\delta_{2}\left(\tau, \tau_{0}\right) / \varepsilon}
\end{array}\right) S\left(\tau_{0}\right)^{-1} \\
& \delta_{1}\left(\tau, \tau_{0}\right)=\int_{\tau_{0}}^{\tau} d_{1}(s) \mathrm{d} s=\frac{1}{2}\left(\tau^{2}-\tau_{0}^{2}\right)-\left(\tau-\tau_{0}\right)+\mathcal{O}\left(\mu^{2}\right) \\
& \delta_{2}\left(\tau, \tau_{0}\right)=\int_{\tau_{0}}^{\tau} d_{2}(s) \mathrm{d} s=(\mathrm{i}-1)\left(\tau-\tau_{0}\right)+\mathcal{O}\left(\mu^{2}\right) .
\end{aligned}
$$

For $\tau>1>\tau_{0}$, however, the solution $s_{2}(\tau)$ necessarily admits a discontinuity of order $\varepsilon \mu$ at the buffer time. A similar calculation as in the previous subsection yields the evolution operator

$$
U\left(\tau, \tau_{0}\right)=S(\tau)\left(\begin{array}{cc}
\mathrm{e}^{\delta_{1}\left(\tau, \tau_{0}\right) / \varepsilon} & \mathcal{O}(\varepsilon \mu) \mathrm{e}^{\left[\delta_{1}(\tau, 1)+\delta_{2}\left(1, \tau_{0}\right)\right] / \varepsilon} \\
0 & \mathrm{e}^{\delta_{2}\left(\tau, \tau_{0}\right) / \varepsilon}
\end{array}\right) S\left(\tau_{0}\right)^{-1}
$$

If the system starts away from the origin at $\tau_{0}<0$, it will follow the origin exponentially closely until a delay time $\hat{\tau}$, which is the first time at which one of the matrix elements becomes of order 1 again. If $\mu=0$, we simply have $\hat{\tau}=2-\tau_{0}$. When $\mu \neq 0$, however, we cannot overlook the interaction between the overdamped and 
the oscillating mode, which takes effectively place at the buffer time $\tau_{\mathrm{b}}=1$, and may cause the system to become unstable at an earlier time. For instance, when $\tau_{0}=-2$, the usual delay time for $\mu=0$ would be $\hat{\tau}=4$, while the effective delay time for $\mu>0$ is $1+\sqrt{6}+\mathcal{O}\left(\mu^{2}\right)$ (Fig. 18b).

A similar phenomenon is observed for the Kobayashi equations (69), only with different values of the cruising, buffer and delay times. The effective value of the bifurcation delay can be shown to depend at leading order in $\varepsilon$ only on the linearization around the origin. It is important, together with nonlinear terms, for the global structure of motion, since it influences the choice of the asymmetric equilibrium the system follows after leaving the origin. In fact, for large amplitude oscillations of the form $\lambda(\tau)=8 \sin (\tau)$, we observed numerically that the Kobayashi equations display chaotic hysteresis just as the rotating pendulum in section 5. This is not really surprising, since even when $\mu=0$, each oscillator is similar to the rotating pendulum of the amplitude of $\lambda$ is large enough. A positive $\mu$, however, will modify the bifurcation delay and the dynamic phases and amplitudes which determine the structure of the Poincaré map.

\section{Acknowledgments}

This work is supported by the Fonds National Suisse de la Recherche Scientifique.

\section{References}

[Ben] E. Benoît (Ed.), Dynamic Bifurcations, Proceeding, Luminy 1990 (Springer-Verlag, Lecture Notes in Mathematics 1493, Berlin, 1991).

[Ber] N. Berglund, Adiabatic Dynamical Systems and Hysteresis, Thesis EPFL no 1800. Available at http://dpwww.epfl.ch/instituts/ipt/berglund/these.html

[BK] N. Berglund, H. Kunz, Chaotic Hysteresis in an Adiabatically Oscillating Double Well, Phys. Rev. Letters 78:1692-1694 (1997).

[GBS] G.H. Goldsztein, F. Broner, S.H. Strogatz, Dynamical Hysteresis without Static Hysteresis: Scaling Laws and Asymptotic Expansions, SIAM J. Appl. Math. 57:1163-1187 (1997).

[HK] J. Hale, H. Koçak, Dynamics and Bifurcations (Springer-Verlag, New York, 1991).

[HL\&] A. Hohl, H.J.C. van der Linden, R. Roy, G. Goldsztein, F. Broner, S.H. Strogatz, Scaling Laws for Dynamical Hysteresis in a Multidimensional Laser System, Phys. Rev. Letters 74:2220-2223 (1995).

[JGRM] P. Jung, G. Gray, R. Roy, P. Mandel, Scaling Law for Dynamical Hysteresis, Phys. Rev. Letters 65:1873-1876 (1990). 
[Ka] K. Kawasaki, Kinetics of Ising Models, in C. Domb, M.S. Green, Phase Transitions and Critical Phenomena, Vol.2 (Academic Press, London, 1972).

[Ko] S. Kobayashi, Two-dimensional Panel Flutter 1. Simply Supported Panel, Trans. Jpn. Soc. Aeronaut. Space Sci. 5:90-102 (1962).

[Ma1] P.A. Martin, Modèles en Mécanique Statistique des Processus Irréversibles (Springer-Verlag, Berlin, 1979).

[Ma2] Ph.A. Martin, On the Stochastic Dynamics of Ising Models, J. Stat. Phys. 16:149-168 (1977).

[MS] W. de Melo, S. van Strien, One-Dimensional Dynamics (Springer, Berlin, 1993).

[MK\&] E.F. Mishchenko, Yu.S. Kolesov, A.Yu. Kolesov, N.Kh. Rozov, Asymptotic Methods in Singularly Perturbed Systems (Consultants Bureau, New York, 1994).

[Ne1] A.I. Neishtadt, Persistence of stability loss for dynamical bifurcations I, Diff. Equ. 23:1385-1391 (1987). Transl. from Diff. Urav. 23:2060-2067 (1987).

[Ne2] A.I. Neishtadt, Persistence of stability loss for dynamical bifurcations II, Diff. Equ. 24:171-176 (1988). Transl. from Diff. Urav. 24:226-233 (1988).

[Ne3] A.I. Neishtadt, On Calculation of Stability Loss Delay Time for Dynamical Bifurcations in D. Jacobnitzer Ed., XI ${ }^{\text {th }}$ International Congress of Mathematical Physics (International Press, Boston, 1995).

[RKP] M. Rao, H.K. Krishnamurthy, R. Pandit, Magnetic hysteresis in two model spin systems, Phys. Rev. B 42:856-884 (1990).

[SE] J.-S. Suen, J.L. Erskine, Magnetic Hysteresis Dynamics: Thin $p(1 \times 1)$ Fe Films on Flat and Stepped W(110), Phys. Rev. Letters 78:3567-3570 (1997).

[TO] T. Tomé, M.J. de Oliveira, Dynamic phase transition in the kinetic Ising model under a time-dependent oscillating field, Phys. Rev. A 41:42514254 (1990).

[Wa] W. Wasow, Asymptotic expansions for ordinary differential equations (Krieger, New York, 1965, 1976). 